\title{
Divergence exponentielle des sous-groupes discrets en rang supérieur
}

Jean-François Quint

Résumé. Soient $G$ un groupe de Lie semi-simple, réel, connexe et de centre fini, a un sousespace de Cartan de l'algèbre de Lie de $G$ et $\mathfrak{a}^{+} \subset \mathfrak{a}$ une chambre de Weyl fermée de $\mathfrak{a}$. Si $\Gamma$ est un sous-groupe discret Zariski dense de $G$, nous lui associons une fonction homogène $\psi_{\Gamma}: \mathfrak{a}^{+} \rightarrow \mathbb{R} \cup\{-\infty\}$ qui généralise l'exposant de convergence de $\Gamma$ considéré en $\mathbb{R}$-rang 1 . Nous montrons alors que cette fonction est concave. Dans un travail ultérieur, nous en déduirons des constructions de généralisations des mesures de Patterson-Sullivan.

Nous démontrons aussi des analogues de nos résultats sur les corps locaux.

Mathematics Subject Classification (2000). Primaire 22E40; Secondaire 53C35.

Mots clés. Lie groups, discrete subgroups, higher rank geometries.

\section{Introduction}

\subsection{Résultats}

Soit $G$ un groupe de Lie semi-simple, réel, connexe et de centre fini. On choisit une involution de Cartan $\tau$ de $G$. On note $K$ le sous-groupe compact maximal de $G$ constitué de l'ensemble des points fixes de $\tau$ et $\mathfrak{a}$ un sous-espace de Cartan de l'algèbre de Lie de $\mathfrak{g}$ tel que, pour $x$ dans $\mathfrak{a}, \tau(\exp x)=\exp (-x)$. Soit $\mathfrak{a}^{+} \subset$ $\mathfrak{a}$ une chambre de Weyl. On dispose alors de la décomposition de Cartan $G=$ $K\left(\exp \mathfrak{a}^{+}\right) K$ et de la projection associée $\mu: G \rightarrow \mathfrak{a}^{+}$.

Si $\Gamma$ est un sous-groupe de $G$, l'étude des propriétés asymptotiques de $\Gamma$ passe par la description de l'ensemble $\mu(\Gamma)$. Dans [4], Y. Benoist a démontré que, si $\Gamma$ est un sous-groupe Zariski dense de $G$, le cône asymptote à l'ensemble $\mu(\Gamma)$ est convexe et d'intérieur non vide. On l'appelle cône limite de $\Gamma$.

Si le $\mathbb{R}$-rang de $G$ est égal à 1 , pour $g$ dans $G$, la donnée de $\mu(g)$ est simplement celle de la distance entre le point fixe $x$ de $K$ dans l'espace symétrique de $G$ et son translaté $g x$ par $g$. Si $\Gamma$ est un sous-groupe discret de $G$, un rôle important est alors joué par l'exposant de convergence de la série de Dirichlet

$$
\sum_{\gamma \in \Gamma} e^{-t d(x, \gamma x)} \quad(t \in \mathbb{R}),
$$


c'est-à-dire par le nombre réel

$$
\limsup _{a \rightarrow \infty}\left(\frac{1}{a} \log (\operatorname{card}\{\gamma \in \Gamma \mid d(x, \gamma x) \leq a\})\right) .
$$

Citons par exemple, la théorie de Patterson-Sullivan introduite dans [14] et [18].

Le but de cet article est la généralisation à la situation de rang supérieur de l'étude de la divergence exponentielle des sous-groupes discrets. On voit alors apparaître un phénomène nouveau de convexité, que nous allons à présent décrire.

Soit $\Gamma$ un sous-groupe discret Zariski dense de $G$. Soit $\|$.$\| une norme (invariante$ par le groupe de Weyl) sur a. Si \|.\| est la norme euclidienne provenant d'une métrique riemannienne $G$-invariante sur l'espace symétrique de $G$, pour tout $g$ dans $G,\|\mu(g)\|$ est la distance entre le point fixe de $K$ et son translaté par $g$.

Pour tout cône ouvert $\mathcal{C}$ de $\mathfrak{a}$, on note $\tau_{\mathcal{C}}$ l'exposant de convergence de la série de Dirichlet

$$
\sum_{\substack{\gamma \in \Gamma \\ \mu(\gamma) \in \mathcal{C}}} e^{-t\|\mu(\gamma)\|} \quad(t \in \mathbb{R})
$$

et, pour $x$ dans $\mathfrak{a}$, on pose

$$
\psi(x)=\|x\| \inf \tau_{\mathcal{C}}
$$

la borne inférieure étant prise sur l'ensemble des cônes ouverts $\mathcal{C}$ de a qui contiennent $x$. La fonction homogène $\psi$ ne dépend pas de la norme choisie. Si $\|$.$\| est$ une norme sur $\mathfrak{a}$, la série de Dirichlet

$$
\sum_{\gamma \in \Gamma} e^{-t\|\mu(\gamma)\|} \quad(t \in \mathbb{R})
$$

a pour exposant de convergence

$$
\sup _{x \in \mathfrak{a} \backslash\{0\}} \frac{\psi(x)}{\|x\|} .
$$

Soit $\rho$ la forme linéaire sur a qui est la somme des racines multipliées par la dimension de leurs espaces poids dans l'algèbre de Lie de $\mathfrak{g}$. Notre principal résultat s'écrit :

Théorème. La fonction $\psi$ est majorée par $\rho$. Elle est concave et semi-continue supérieurement. L'ensemble

$$
\{x \in \mathfrak{a} \mid \psi(x)>-\infty\}
$$

est exactement le cône limite de $\Gamma$. De plus, $\psi$ est positive sur le cône limite de $\Gamma$ et strictement positive sur son intérieur relatif.

Dans [17], nous appliquerons ce théorème à la construction de généralisations des mesures de Patterson-Sullivan. Ce problème avait déjà été considéré par $\mathrm{P}$. Albuquerque dans [1]. En $\mathbb{R}$-rang 1, c'est l'étude de ces mesures qui permet, sous 
certaines hypothèses, d'obtenir des équivalents des fonctions orbitales de comptage pour l'action de $\Gamma$ dans l'espace symétrique de $G$. Concernant les questions de comptage, nos méthodes permettent de montrer :

Proposition. Soit \|.\| une norme sur a. Alors

$$
\frac{1}{a} \log (\operatorname{card}\{\gamma \in \Gamma \mid\|\mu(\gamma)\| \leq a\})
$$

admet une limite $\tau \in \mathbb{R}_{+}^{*}$ quand a tend vers $\infty$ et l'on a :

$$
\operatorname{card}\{\gamma \in \Gamma \mid\|\mu(\gamma)\| \leq a\} \underset{a \rightarrow \infty}{=} O\left(a^{r-1} e^{a \tau}\right)
$$

oùr est le $\mathbb{R}$-rang de $G$.

En d'autres termes, en appliquant ce résultat aux normes euclidiennes invariantes par le groupe de Weyl :

Corollaire. Soit $X$ l'espace symétrique de $G$, muni d'une métrique riemannienne $G$-invariante. Alors, il existe $\tau>0$ tel que, pour tous $x, y$ dans $X$, on ait:

$$
\frac{1}{a} \log (\operatorname{card}\{\gamma \in \Gamma \mid d(x, \gamma y) \leq a\}) \underset{a \rightarrow \infty}{\longrightarrow} \tau
$$

et

$$
\operatorname{card}\{\gamma \in \Gamma \mid d(x, \gamma y) \leq a\} \underset{a \rightarrow \infty}{=} O\left(a^{r-1} e^{a \tau}\right)
$$

\subsection{Structure des démonstrations}

La démonstration du théorème s'effectue en deux étapes.

Dans la première partie du texte, on établit :

Proposition. Soit $\Gamma$ un sous-groupe discret Zariski dense de $G$. Il existe une application $\pi: \Gamma \times \Gamma \rightarrow \Gamma$ ayant les propriétés suivantes :

(i) il existe un réel $\kappa \geq 0$ tel que, pour tous $\gamma_{1}, \gamma_{2}$ dans $\Gamma$,

$$
\left\|\mu\left(\pi\left(\gamma_{1}, \gamma_{2}\right)\right)-\mu\left(\gamma_{1}\right)-\mu\left(\gamma_{2}\right)\right\| \leq \kappa .
$$

(ii) pour tout réel $R \geq 0$, il existe une partie finie $H$ de $\Gamma$ telle que, pour $\gamma_{1}, \gamma_{2}, \gamma_{1}^{\prime}, \gamma_{2}^{\prime}$ dans $\bar{\Gamma}$, avec $\left\|\mu\left(\gamma_{1}\right)-\mu\left(\gamma_{1}^{\prime}\right)\right\| \leq R$ et $\left\|\mu\left(\gamma_{2}\right)-\mu\left(\gamma_{2}^{\prime}\right)\right\| \leq R$,

$$
\pi\left(\gamma_{1}, \gamma_{2}\right)=\pi\left(\gamma_{1}^{\prime}, \gamma_{2}^{\prime}\right) \Rightarrow\left(\gamma_{1}^{\prime} \in \gamma_{1} H \text { et } \gamma_{2}^{\prime} \in H \gamma_{2}\right) \text {. }
$$

Une telle application $\pi$ sera, dans la suite du texte, appelée produit générique dans $\Gamma$. L'idée de la preuve est d'écrire $\pi\left(\gamma_{1}, \gamma_{2}\right)=\gamma_{1} f \gamma_{2}$ où $f$ est choisi dans une partie finie $F$ de $\Gamma$, de façon à éliminer les problèmes qui se posent quand 
$\gamma_{1}$ est proche de $\gamma_{2}^{-1}$. La partie $F$ sera construite en utilisant un résultat de $\mathrm{H}$. Abels, G.-A. Margulis et G.-A. Soifer, le lemme 2.3.4, dont nous redonnerons la démonstration. La vérification du point (i) de la proposition s'effectue en estimant la norme de $\gamma_{1} f \gamma_{2}$ dans suffisament de représentations de $G$, au lemme 2.3.3. Le point délicat est la vérification de la partie (ii). Sa démonstration s'inspire de phénomènes de géométrie dans $G / K$ liés à l'existence en rang supérieur d'analogues du fameux lemme des ombres de Sullivan ([18]). On définit, pour $g$ dans $G$, une partie $B_{g}^{\varepsilon}$ de la variété des drapeaux de $G$ de sorte que $g B_{g}^{\varepsilon}$ joue le rôle des ombres de [18] et on montre un analogue du lemme des ombres, la proposition 2.3.7. Reste alors à vérifier que, sous les hypothèses que nous aurons faites, les ombres $\gamma_{1} B_{\gamma_{1}}^{\varepsilon}$ et $\gamma_{1} f \gamma_{2} B_{\gamma_{1} f \gamma_{2}}^{\varepsilon}$ se rencontrent, c'est ce qui est fait dans la démonstration du lemme 2.3.8.

Dans la deuxième partie on déduit de l'existence d'un produit générique dans $\Gamma$ qu'il existe des réels $\alpha, \beta, \gamma>0$ tels que, pour $x, y$ dans $\mathfrak{a}$,

$$
\operatorname{card}\left(\Gamma \cap \mu^{-1}(b(x+y, \alpha))\right) \geq \gamma \operatorname{card}\left(\Gamma \cap \mu^{-1}(b(x, \beta))\right) \operatorname{card}\left(\Gamma \cap \mu^{-1}(b(y, \beta))\right),
$$

et l'on démontre la concavité de $\psi$ à partir de cette seule propriété de l'ensemble $\mu(\Gamma)$.

Enfin, dans une troisième partie, nous terminerons la démonstration précise du théorème.

\subsection{Corps locaux}

Dans l'esprit de [4], nous démontrerons des analogues des résultats ci-dessus pour les groupes semi-simples définis sur un corps valué localement compact. Nous utiliserons les analogues des décompositions de Cartan et d'Iwasawa pour ces groupes, établis par F. Bruhat et J. Tits dans [8] et [9]. Nous renvoyons le lecteur à [20], pour un résumé de cette théorie.

J'ai bénéficié, pour l'élaboration de ce travail, des remarques et des suggestions d'Yves Benoist. Je tiens ici à l'en remercier.

\section{Produit générique}

Soit $\mathbb{K}$ un corps local : $\mathbb{K}$ est soit $\mathbb{R}$ ou $\mathbb{C}$, soit une extension finie de $\mathbb{Q}_{p}$, pour un entier premier $p$, soit le corps des fractions $\mathbb{F}_{q}((T))$ de l'anneau des séries formelles sur le corps fini à $q$ éléments.

Si $\mathbb{K}$ est $\mathbb{R}$ ou $\mathbb{C}$, on le munit de la valeur absolue usuelle et on pose $q=e$, $u=e^{-1}$ et pour tout $x \neq 0$ dans $\mathbb{K}, \omega(x)=-\log |x|$.

Si $\mathbb{K}$ est non-archimédien, on note $\mathcal{O}$ l'anneau de valuation de $\mathbb{K}, \mathfrak{m}$ l'idéal maximal de $\mathcal{O}, k=\mathcal{O} / \mathfrak{m}$ le corps résiduel de $\mathbb{K}, q$ le cardinal de $k$ et $u$ une uniformisante de $\mathbb{K}$, i.e. un élément de $\mathfrak{m} \backslash \mathfrak{m}^{2}$; on note $\omega$ la valuation de $\mathbb{K}$ telle 
que $\omega(u)=1$ et on munit $\mathbb{K}$ de la valeur absolue $x \mapsto q^{-\omega(x)}$.

Étant donnée une extension algébrique de $\mathbb{K}$, on la munit de l'unique valeur absolue prolongeant celle de $\mathbb{K}$.

Soit $(X, d)$ un espace métrique. Pour tout $\varepsilon>0$, pour toute partie $Y$ de $X$, on note :

$$
b(Y, \varepsilon)=\{x \in X \mid d(x, Y) \leq \varepsilon\} \text { et } B(Y, \varepsilon)=\{x \in X \mid d(x, Y) \geq \varepsilon\} .
$$

Pour toutes parties $Y$ et $Z$ de $X$, on note:

$$
d(Y, Z)=\inf _{(y, z) \in Y \times Z} d(y, z) \text { et } \delta(Y, Z)=\sup _{y \in Y} d(y, Z) .
$$

Pour tout ensemble $X$ et pour tout $x$ dans $X$, on note $\delta_{x}$ la mesure de Dirac en $x$.

Si $t$ est un nombre réel, on note $[t]$ sa partie entière.

\subsection{Algèbre linéaire normée}

Nous démontrons ici l'ensemble des résultats d'algèbre linéaire qui seront utilisés dans ce texte. Ils seront ensuite réinterprétés dans les groupes réductifs, à travers leurs représentations linéaires.

Soit $V$ un $\mathbb{K}$-espace vectoriel de dimension finie $m$. On munit $\mathbb{P}(V)$ de la topologie quotient de celle de $V-\{0\}$ : c'est un espace topologique compact.

\subsubsection{Rayon spectral et proximalité}

Soit $f$ un endomorphisme de $V$. On note $\lambda_{1}(f)$ le rayon spectral de $f$, c'està-dire le plus grand des modules des valeurs propres de $f$. On note $V_{f}^{+}$le plus grand sous-espace vectoriel $f$-stable de $V$ où toutes les valeurs propres de $f$ sont de module $\lambda_{1}(f)$ et $V_{f}^{<}$l'unique supplémentaire $f$-stable de $V_{f}^{+}$.

Munissons $V$ d'une norme. On a la formule du rayon spectral :

$$
\forall f \in \mathcal{L}(V) \quad\left\|f^{n}\right\|^{\frac{1}{n}} \underset{n \rightarrow \infty}{\longrightarrow} \lambda_{1}(f) .
$$

Un endomorphisme $f \neq 0$ de $V$ est dit proximal dans $\mathbb{P}(V)$ si et seulement si $f$ possède une unique valeur propre de module maximal et qu'elle est de multiplicité 1 , i.e. si et seulement si $\operatorname{dim} V_{f}^{+}=1$. Cette valeur propre appartient alors à $\mathbb{K}$.

Soit $f$ un endomorphisme non nul de $V$. Alors $f$ est proximal dans $\mathbb{P}(V)$ si et seulement si $f$ possède un point fixe attracteur dans $\mathbb{P}(V)$. Ce point fixe est alors $V_{f}^{+}$.

\subsubsection{Bonnes normes et bonnes sommes directes}

Une norme sur $V$ est une application $\|\|:. V \rightarrow \mathbb{R}_{+}$vérifiant les axiomes usuels :

(i) $\forall v \in V \quad\|v\|=0 \Leftrightarrow v=0$. 
(ii) $\forall \lambda \in \mathbb{K} \quad \forall v \in V \quad\|\lambda v\|=|\lambda|\|v\|$.

(iii) $\forall v, w \in V \quad\|v+w\| \leq\|v\|+\|w\|$.

Si $\mathbb{K}$ est $\mathbb{R}$ (resp. $\mathbb{C}$ ), on dit qu'une norme sur $V$ est une bonne norme si et seulement si elle est induite par un produit scalaire euclidien (resp. un produit scalaire hermitien). Si $V$ est muni d'une bonne norme, on dit qu'une somme directe $V=V_{1} \oplus V_{2}$ est une bonne somme directe si et seulement si elle est orthogonale pour le produit scalaire.

Si $\mathbb{K}$ est non archimédien, on dit qu'une norme sur $V$ est une bonne norme si et seulement si elle est ultramétrique, c'est-à-dire si et seulement si, pour tous $v, w$ dans $V$, on a $\|v+w\| \leq \max (\|v\|,\|w\|)$. Si $V$ est muni d'une bonne norme, on dit qu'une somme directe $V=V_{1} \oplus V_{2}$ est une bonne somme directe si et seulement si, pour tout $v=v_{1}+v_{2}$ dans $V$, avec $v_{1}$ dans $V_{1}$ et $v_{2}$ dans $V_{2}$, on a :

$$
\|v\|=\max \left(\left\|v_{1}\right\|,\left\|v_{2}\right\|\right) .
$$

Supposons dorénavant $V$ muni d'une bonne norme. Donnons une caractérisation des bonnes sommes directes; c'est une généralisation d'un exercice classique de géométrie euclidienne :

Lemme 2.1.1. Soit $V=V_{1} \oplus V_{2}$ une somme directe dans $V$. Elle est bonne si et seulement si ses projecteurs sont de norme 1 .

Démonstration. Soit $p$ le projecteur sur $V_{1}$ parallèlement à $V_{2}$. Si la somme directe est bonne, on a $\|p\|=1$. Réciproquement, supposons que $p$ est de norme 1 .

Supposons que $\mathbb{K}$ est $\mathbb{R}$ ou $\mathbb{C}$. Soit $v$ dans $V_{2}^{\perp}$. Soit $w=v-p(v)$. Alors $v$ et $w$ sont orthogonaux et $p(v)=v-w$. Par conséquent, on a :

$$
\|v\| \geq\|p(v)\|=\sqrt{\|v\|^{2}+\|w\|^{2}} \geq\|v\|
$$

et, donc, $\|p(v)\|=\|v\|$, ou encore $w=0$. Il vient $V_{2}^{\perp} \subset V_{1}$ et, comme ces deux espaces ont même dimension, $V_{2}^{\perp}=V_{1}$, ce qu'il fallait démontrer.

Supposons que $\mathbb{K}$ est non-archimédien. Remarquons que l'on a $\|1-p\| \leq$ $\max (1,\|p\|) \leq 1$. Pour tout $v$ dans $V$, il vient :

$$
\|v\|=\|p(v)+(1-p)(v)\| \leq \max (\|p(v)\|,\|(1-p)(v)\|) \leq\|v\|
$$

et, donc, $\|v\|=\max (\|p(v)\|,\|(1-p)(v)\|)$, ce qu'il fallait démontrer.

Il existe une unique bonne norme sur $\wedge^{2} V$ telle que, pour toute bonne somme directe $V_{1} \oplus V_{2} \subset V$ la somme directe $\left(\wedge^{2} V_{1}\right) \oplus\left(V_{1} \wedge V_{2}\right) \oplus\left(\wedge^{2} V_{2}\right) \subset \wedge^{2} V$ soit bonne et que, pour $v, w$ dans $V$, si $\mathbb{K} v$ et $\mathbb{K} w$ sont en bonne somme directe, on ait $\|v \wedge w\|=\|v\|\|w\|$. Alors, l'application

$$
\begin{aligned}
(V-\{0\})^{2} & \rightarrow \mathbb{R}_{+} \\
(v, w) & \mapsto \frac{\|v \wedge w\|}{\|v\|\|w\|}
\end{aligned}
$$


factorise à travers une distance sur $\mathbb{P}(V)$, qui y induit sa topologie usuelle. C'est un résultat classique si $\mathbb{K}$ est $\mathbb{R}$ ou $\mathbb{C}$. Le cas général est traité dans [15]. On munira toujours l'espace projectif d'un espace vectoriel bien normé de cette distance. Si le dual $V^{*}$ de $V$ est muni de la bonne norme duale de celle de $V$, pour tous $v \neq 0$ dans $\mathbb{P}(V)$ et $\varphi \neq 0$ dans $\mathbb{P}\left(V^{*}\right)$, on a :

$$
d\left(\mathbb{K} v, \varphi^{\perp}\right)=\frac{|\varphi(v)|}{\|\varphi\|\|v\|}=d\left(\mathbb{K} \varphi, v^{\perp}\right) .
$$

Nous utiliserons :

Lemme 2.1.2. Soit $\varepsilon>0$. Soient $V=V_{1} \oplus V_{2}$ une bonne somme directe et $p$ le projecteur sur $V_{1}$ parallèlement à $V_{2}$. Pour tout $v$ dans $V-\{0\}$ avec $d\left(\mathbb{K} v, \mathbb{P}\left(V_{2}\right)\right) \geq$ $\varepsilon$, on $a\|p(v)\| \geq \varepsilon\|v\|$.

Démonstration. On peut, bien sûr, supposer $v \notin V_{1}$. Alors, soient $v_{1}$ et $v_{2}$ les composantes de $v$ sur $V_{1}$ et $V_{2}$. On a $d\left(\mathbb{K} v, \mathbb{K} v_{2}\right) \geq \varepsilon$. Or

$$
d\left(\mathbb{K} v, \mathbb{K} v_{2}\right)=\frac{\left\|v \wedge v_{2}\right\|}{\|v\|\left\|v_{2}\right\|}=\frac{\left\|v_{1} \wedge v_{2}\right\|}{\|v\|\left\|v_{2}\right\|}=\frac{\left\|v_{1}\right\|}{\|v\|},
$$

d'où le résultat.

\subsubsection{Semi-similitudes}

Nous étudions ici une classe particulière d'endomorphismes d'un $\mathbb{K}$-espace vectoriel normé.

On dit qu'un endomorphisme $f$ de $V$ est une similitude si et seulement s'il existe un réel $\lambda \geq 0$ tel que, pour tout $v$ dans $V$, on ait :

$$
\|f(v)\|=\lambda\|v\| \text {. }
$$

On dit alors que $\lambda$ est le rapport de $f$. On dit que $f$ est une semi-similitude si et seulement s'il existe une bonne somme directe $V=V_{1} \oplus \ldots \oplus V_{k}$ telle que, pour tout $i$ dans $\llbracket 1, k \rrbracket, f$ stabilise $V_{i}$ et induise sur $V_{i}$ une similitude de rapport $\lambda_{i}$. Dans ce cas, on peut supposer que l'on a :

$$
\lambda_{1}>\ldots>\lambda_{k}
$$

On a alors, pour tout $v$ dans $V, \lambda_{k}\|v\| \leq\|f v\| \leq \lambda_{1}\|v\|$. En particulier, $\lambda_{1}$ est à la fois la norme et le rayon spectral de $f$.

Si $\mathbb{K}$ est $\mathbb{R}$ ou $\mathbb{C}$, une semi-similitude est simplement un endomorphisme normal de $V$.

Lemme 2.1.3. Soit $f$ une semi-similitude. Soit $V=V_{1} \oplus \ldots \oplus V_{k}$ une bonne somme directe dans $V$ telle que, pour tout $i$ dans $\llbracket 1, k \rrbracket$, $f$ laisse stable $V_{i}$ et induise sur $V_{i}$ une similitude de rapport $\lambda_{i}$. Supposons que l'on a $\lambda_{1}>\ldots>\lambda_{k}$.

(i) Soit $W$ un sous-espace vectoriel de $V$ stable par $f$. Alors on a $W=(W \cap$ $\left.V_{1}\right) \oplus \ldots \oplus\left(W \cap V_{k}\right)$ et, en particulier, la restriction de $f \grave{a} W$ est une semi-similitude. 
(ii) Soit $V=W_{1} \oplus \ldots \oplus W_{l}$ une autre somme directe, non nécessairement bonne, telle que, pour tout $j$ dans $\llbracket 1, l \rrbracket$, $f$ laisse stable $W_{j}$ et induise sur $W_{j}$ une similitude de rapport $\mu_{j}$ et que l'on ait $\mu_{1}>\ldots>\mu_{l}$. Alors $k=l$ et, pour tout $i$ dans $\llbracket 1, k \rrbracket, W_{i}=V_{i}$ et $\lambda_{i}=\mu_{i}$.

Démonstration. Pour tout entier $n$, posons

$$
h_{n}=-\left[\frac{\log \left(\left\|f^{n}\right\|\right)}{\log q}\right] \text { et } p_{n}=\frac{1}{u^{h_{n}}} f^{n} .
$$

On a, pour tout $n$ dans $\mathbb{N}$,

$$
1 \leq\left\|p_{n}\right\| \leq q
$$

On fixe une valeur d'adhérence $p$ dans $\mathcal{L}(V)$ de la suite $\left(p_{n}\right)_{n \in \mathbb{N}}$.

Pour tout $v$ dans $V_{1}$, le vecteur $p(v)$ appartient à $V_{1}$ et $\|p(v)\|=\|p\|\|v\|$. En particulier, la restriction de $p$ à $V_{1}$ est un automorphisme. Par ailleurs, pour tout $v$ dans $V_{2} \oplus \ldots \oplus V_{k}$, on a $p(v)=0$.

Soit $W$ un sous-espace vectoriel de $V$ stable par $f$. Alors, $W$ est stable par $p$. En particulier, $W \cap V_{1}$ est stable par $p$ et, donc, par l'inverse de la restriction de $p$ à $V_{1}$.

Soit $v$ dans $W$. Écrivons $w=v_{1}+v^{\prime}$ avec $v_{1}$ dans $V_{1}$ et $v^{\prime}$ dans $V_{2} \oplus \ldots \oplus V_{k}$. On a :

$$
p(v)=p\left(v_{1}\right) \in W \cap V_{1}
$$

et, donc,

$$
v_{1} \in W
$$

Il vient :

$$
W=\left(W \cap V_{1}\right) \oplus\left(W \cap\left(V_{2} \oplus \ldots \oplus V_{k}\right)\right)
$$

d'où la première propriété par récurrence.

La seconde en est une conséquence.

Soit $f$ une semi-similitude. On note $V_{f}^{M}$ le plus grand sous-espace vectoriel stable par $f$ où $f$ induise une similitude de rapport $\|f\|$ et $V_{f}^{m}$ son unique supplémentaire stable par $f$. La somme directe $V=V_{f}^{M} \oplus V_{f}^{m}$ est bonne. Une semi-similitude $f$ est proximale si et seulement si $\operatorname{dim} V_{f}^{M}=1$ et, alors, on a $V_{f}^{+}=V_{f}^{M}$ et $V_{f}^{<}=V_{f}^{m}$.

\subsubsection{Propriétés des semi-similitudes}

Nous effectuons ici des contrôles uniformes sur l'action des semi-similitudes dans $\mathbb{P}(V)$ qui seront utilisés pour la construction du produit générique, à la section 2.3 .

Commençons par remarquer que beaucoup de vecteurs permettent d'estimer la norme d'une semi-similitude : 
Lemme 2.1.4. Soient $f$ une semi-similitude de $V$ et $\varepsilon>0$. Pour tout vecteur non nul $v$ de $V$, sid $\left(\mathbb{K} v, \mathbb{P}\left(V_{f}^{m}\right)\right) \geq \varepsilon$, alors on a $\|f v\| \geq \varepsilon\|f\|\|v\|$.

Démonstration. Écrivons $v=v_{1}+v_{2}$ avec $v_{1}$ dans $V_{f}^{M}$ et $v_{2}$ dans $V_{f}^{m}$. D'après le lemme 2.1.2, on a $\left\|v_{1}\right\| \geq \varepsilon\|v\|$ et, donc,

$$
\|f v\| \geq\left\|f v_{1}\right\|=\|f\|\left\|v_{1}\right\| \geq \varepsilon\|f\|\|v\| .
$$

On a aussi une information sur l'action des semi-similitudes sur $\mathbb{P}(V)$ en termes de métrique :

Lemme 2.1.5. Soient $f$ une semi-similitude de $V$ et $\varepsilon>0$. La restriction de $f \grave{a}$ $B\left(\mathbb{P}\left(V_{f}^{m}\right), \varepsilon\right)$ est $\frac{1}{\varepsilon^{2}}$-lipschitzienne.

Démonstration. Remarquons que, comme $f$ est une semi-similitude, on a $\left\|\wedge^{2} f\right\| \leq$ $\|f\|^{2}$. Donnons-nous alors deux vecteurs non nuls $v$ et $w$ avec $d\left(\mathbb{K} v, \mathbb{P}\left(V_{f}^{m}\right)\right) \geq \varepsilon$ et $d\left(\mathbb{K} w, \mathbb{P}\left(V_{f}^{m}\right)\right) \geq \varepsilon$. D'après le lemme 2.1.4, on a $\|f v\| \geq \varepsilon\|f\|\|v\|$ et $\|f v\| \geq$ $\varepsilon\|f\|\|v\|$. Il vient :

$$
\begin{aligned}
d(\mathbb{K} f v, \mathbb{K} f w)=\frac{\|(f v) \wedge(f w)\|}{\|f v\|\|f w\|} & \leq \frac{\|f\|^{2}\|v \wedge w\|}{\|f v\|\|f w\|} \\
& \leq \frac{1}{\varepsilon^{2}} \frac{\|v \wedge w\|}{\|v\|\|w\|}=\frac{1}{\varepsilon^{2}} d(\mathbb{K} v, \mathbb{K} w) .
\end{aligned}
$$

Le lemme suivant et notre généralisation du lemme des ombres serviront de base au contrôle de distance dans la construction du produit générique :

Lemme 2.1.6. Soient $r>0$ et $\varepsilon>0$. Il existe un réel $\eta>0$ tel que pour toute semi-similitude $f$ de $V$, pour tout hyperplan $W$ de $V$ avec $\delta\left(\mathbb{P}\left(V_{f}^{M}\right), \mathbb{P}(W)\right) \geq r$, on ait :

$$
f^{-1} b(\mathbb{P}(W), \eta) \subset b\left(\mathbb{P}\left(f^{-1} W\right), \varepsilon\right) .
$$

Démonstration. Soit $f$ une semi-similitude de $V$. Alors, son adjoint $f^{*}$ est une semi-similitude de $V^{*}$. Soit $\varphi$ une forme linéaire non nulle de $V$. On a $f^{-1}\left(\varphi^{\perp}\right)=$ $\left(f^{*}(\varphi)\right)^{\perp}$. Supposons que $\delta\left(\mathbb{P}\left(V_{f}^{M}\right), \mathbb{P}\left(\varphi^{\perp}\right)\right) \geq r$. Alors, comme, pour $v$ dans $V_{f}^{M},\left(V^{*}\right)_{f^{*}}^{m} \subset v^{\perp}$, on a

$$
d\left(\mathbb{K} \varphi, \mathbb{P}\left(\left(V^{*}\right)_{f^{*}}^{m}\right)\right) \geq r .
$$

Soit $0<\eta \leq \frac{r}{2}$. Soit $v$ un vecteur non nul de $V$ et supposons que l'on a $d\left(\mathbb{K} v, \mathbb{P}\left(\varphi^{\perp}\right)\right) \leq \eta$. On a $d\left(\mathbb{K} \varphi, \mathbb{P}\left(v^{\perp}\right)\right) \leq \eta$ et, donc, d'après le lemme 2.1.5, 
on a $d\left(\mathbb{K} f^{*}(\varphi), \mathbb{P}\left(f^{*}\left(v^{\perp}\right)\right)\right) \leq \frac{4 \eta}{r^{2}}$. Comme $f^{*}\left(v^{\perp}\right)=\left(f^{-1}(v)\right)^{\perp}$, il vient,

$$
f^{-1} b\left(\varphi^{\perp}, \eta\right) \subset b\left(f^{-1}\left(\varphi^{\perp}\right), \frac{4 \eta}{r^{2}}\right),
$$

d'où le résultat.

\subsection{Groupes réductifs}

On fixe un $\mathbb{K}$-groupe réductif connexe $\mathbf{G}$. On note $G$ le groupe de ses $\mathbb{K}$-points. Nous introduisons ici le vocabulaire concernant $G$ et ses décompositions qui sera utilisé dans la suite du texte. Le lecteur trouvera plus de précisions, pour la théorie générale des groupes réductifs, dans [6] et [12], pour la théorie sur $\mathbb{R}$ ou $\mathbb{C}$, dans [10] et [11] et, pour la théorie sur des corps non-archimédiens, dans [8], [9] et [20].

\subsubsection{Système de racines et chambre de Weyl}

Pour tout $\mathbb{K}$-groupe $\mathbf{H}$, on note $\mathrm{X}(\mathbf{H})$ le groupe de ses caractères rationnels.

On note $r$ le $\mathbb{K}$-rang de $\mathbf{G}$. On fixe un tore $\mathbb{K}$-déployé maximal $\mathbf{A}$ de $\mathbf{G}$ et on note $A$ le groupe de ses $\mathbb{K}$-points. On note $\mathbf{Z}$ le centralisateur de $\mathbf{A}$ dans $\mathbf{G}$ et $Z$ le groupe de ses $\mathbb{K}$-points. Le groupe $\mathrm{X}(\mathbf{A})$ est un groupe abélien libre de rang $r$. L'homomorphisme de restriction identifie $\mathbf{X}(\mathbf{Z})$ à un sous-groupe d'indice fini de $\mathrm{X}(\mathbf{A})$. On note $E^{*}$ le $\mathbb{R}$-espace vectoriel $\mathbb{R} \otimes_{\mathbb{Z}} \mathrm{X}(\mathbf{A})$ et $E$ son dual. Pour tout $\chi$ dans $\mathrm{X}(\mathbf{A})$, on note $\chi^{\omega}$ la forme linéaire associée sur $E$.

Soit $\mathfrak{g}$ l'algèbre de Lie de $\mathbf{G}$. Soit $\Sigma$ l'ensemble des racines de $\mathbf{A}$ dans $\mathfrak{g}$. Alors $\Sigma^{\omega}$ est un système de racines dans $E^{*}$. On choisit dans $\Sigma$ un système de racines positives $\Sigma^{+}$et on note $\Pi$ la base de $\Sigma$ associée à ce choix.

On note $E^{+}$et $E^{++}$les chambres de Weyl positive et strictement positive de $\Sigma^{+}$dans $E^{+}$. On munit $E$ de l'ordre associé à $E^{+}$: si $x$ et $y$ sont deux vecteurs de $E$, on a $x \geq y$ si et seulement si $x-y$ appartient à $E^{+}$. Plus généralement, pour tous $x, y$ dans $E$, pour tout $C \geq 0$, on note $x \geq_{C} y$ si et seulement si

$$
\forall \alpha \in \Pi \quad \alpha^{\omega}(x-y) \geq-C .
$$

On note $W$ le groupe de Weyl de $\Sigma$ : il s'identifie au quotient du normalisateur de $A$ dans $G$ par $Z$. Pour tout $\alpha$ dans $\Sigma$, on note $\sigma_{\alpha} \in W$ la réflexion associée. On note $w_{0}$ le plus long élément de $W$ : c'est l'unique élément de $W$ qui envoie $E^{+}$sur $-E^{+}$. On appelle $\iota=-w_{0}$ l'involution d'opposition de $E^{+}$. On note $E_{S}$ l'unique supplémentaire $W$-stable de l'espace $E^{W}$ des points fixes de $W$ dans $E$ et $\left(\varpi_{\alpha}\right)_{\alpha \in \Pi}$ la famille des poids fondamentaux de $\Pi$, avec la convention $\left(\varpi_{\alpha}\right)_{\mid E^{W}}=0$, pour $\alpha$ dans $\Pi$.

Pour tout $z$ dans $Z$, on note $\nu(z)$ l'unique vecteur de $E$ tel que, pour tout $\chi$ dans $\mathrm{X}(\mathbf{Z})$, on ait :

$$
\chi^{\omega}(\nu(z))=-\omega(\chi(z))
$$


L'application $\nu$ est un homomorphisme de groupes de $Z$ dans $E$. Si $\mathbb{K}$ est $\mathbb{R}$ ou $\mathbb{C}$, l'application $\nu$ est surjective. Si $\mathbb{K}$ est non-archimédien, l'image de $\nu$ est un réseau stable par l'action de $W$ dans $E$. On note $Z^{+}=\nu^{-1}\left(E^{+}\right)$.

Pour tout $\alpha$ dans $\Sigma$, on note $m_{\alpha}$ la dimension de l'espace poids de $\alpha$ dans $\mathfrak{g}$ et on pose $\rho=\prod_{\alpha \in \Sigma^{+}} \alpha^{m_{\alpha}}$.

Dorénavant, on considèrera tout caractère rationnel de $\mathbf{A}$ comme une forme linéaire sur $E$. On fixe une partie $X_{C}$ de $\mathrm{X}(\mathbf{Z})$ qui engendre $\left(E^{*}\right)^{W}$

On fixe un produit scalaire $W$-invariant $(.,$.$) sur E$. On note $\|\cdot\|$ la norme euclidienne associée.

\subsubsection{Facettes}

On note $\mathbf{P}_{\Pi}$ le $\mathbb{K}$-sous-groupe parabolique minimal de $\mathbf{G}$ associé au choix de $\mathbf{A}$ et de $\Sigma^{+}$.

Soit $\theta \subset \Pi$. On note $\theta^{c}$ le complémentaire de $\theta$ dans $\Pi$.

On note

$$
E_{\theta}=\bigcap_{\alpha \in \theta^{c}} \operatorname{ker} \alpha, E_{\theta}^{+}=E_{\theta} \cap E^{+} \text {et } E_{\theta}^{++}=E_{\theta}^{+}-\left(\bigcup_{\tau \varsubsetneqq \theta} E_{\tau}^{+}\right) .
$$

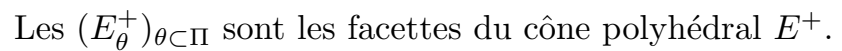

On note $W_{\theta}$ le fixateur de $E_{\theta}$ dans $W$ : c'est le sous-groupe de $W$ engendré par les réflexions associées aux éléments de $\theta^{c}$. On note $p_{\theta}$ l'unique projecteur (orthogonal) $W_{\theta}$-invariant de $E$ dans $E_{\theta}$. Nous aurons à utiliser :

Lemme 2.2.1. Pour tout $x$ dans $E$, pour tout $y$ dans $E_{\theta}$, on $a$ :

$$
\left(p_{\theta}(x)=y\right) \Leftrightarrow\left(\forall \chi \in X_{C} \cup\left\{\varpi_{\alpha} \mid \alpha \in \theta\right\} \quad \chi(x)=\chi(y)\right) .
$$

Démonstration. Nous utilisons ici librement les résultats de [7, 1.10].

Il s'agit de montrer que l'on a :

$$
\operatorname{ker} p_{\theta}=E_{S} \cap \bigcap_{\alpha \in \theta} \operatorname{ker} \varpi_{\alpha} .
$$

Soit $p_{\theta}^{*}$, l'adjoint de $p_{\theta}$. Alors, comme l'image de $p_{\theta}$ est $E_{\theta}, p_{\theta}^{*}$ est un projecteur orthogonal de noyau $\bigoplus_{\alpha \in \theta^{c}} \mathbb{R} \alpha$ et, donc, d'image

$$
\left(\bigoplus_{\alpha \in \theta^{c}} \mathbb{R} \alpha\right)^{\perp}=\bigoplus_{\alpha \in \theta} \mathbb{R} \varpi_{\alpha} \oplus\left(E^{*}\right)^{W},
$$

d'où le résultat.

On note $\mathbf{A}_{\theta}$ la composante Zariski connexe de $\bigcap_{\alpha \in \theta^{c}} \operatorname{ker} \alpha$ dans $\mathbf{A}$. Soit $\mathbf{L}_{\theta}$ le centralisateur de $\mathbf{A}_{\theta}$ dans $\mathbf{G}$ : c'est un $\mathbb{K}$-groupe réductif connexe. On note $L_{\theta}$ le groupe de ses $\mathbb{K}$-points. On note $\mathbf{P}_{\theta}$ le $\mathbb{K}$-groupe $\mathbf{L}_{\theta} \mathbf{P}_{\Pi}$ et $P_{\theta}$ le groupe de ses $\mathbb{K}$-points. De même, on note $\mathbf{P}_{\theta}^{\vee}$ le $\mathbb{K}$-sous-groupe parabolique de $\mathbf{G}$ opposé à $\mathbf{P}_{\theta}$ par rapport à $\mathbf{A}$ et $P_{\theta}^{\vee}$ le groupe de ses $\mathbb{K}$-points. 


\subsubsection{Représentations de G}

Soit $(\rho, V)$ une représentation rationnelle irréductible de dimension finie de $\mathbf{G}$.

On appelle poids restreints de $\rho$ les poids rationnels de la représentation $\rho_{\mid \mathbf{A}}$. D'après $[19,7.2]$, l'ensemble des poids restreints possède un plus grand élément $\chi$ pour l'ordre associé à $\Pi$ sur $E^{*}$. On dit que $\chi$ est le plus haut poids restreint de $\rho$. Les autres poids restreints sont de la forme $\chi-\sum_{\alpha \in \Pi} n_{\alpha} \alpha$ avec, pour tout $\alpha$ dans $\Pi, n_{\alpha} \in \mathbb{N}$. On note $V_{\Pi}^{+}$l'espace poids associé à $\chi$ et $V_{\Pi}^{<}$l'unique supplémentaire $A$-stable de $V_{\Pi}^{+}$.

D'après [19], on a :

Proposition 2.2.2. (Tits) Il existe une famille de représentations rationnelles irréductibles $\left(\rho_{\alpha}, V_{\alpha}\right)_{\alpha \in \Pi}$ de $\mathbf{G}$ telles que, pour tout $\alpha$ dans $\Pi$, le plus haut poids restreint $\chi_{\alpha}$ de $\left(\rho_{\alpha}, V_{\alpha}\right)$ soit un multiple du poids fondamental associé à $\alpha$ et que $\operatorname{dim} V_{\alpha, \Pi}^{+}=1$.

Dorénavant, on fixe une telle famille de représentations. D'après le lemme 2.2.1, on a :

Lemme 2.2.3. Pour tout $\theta \subset \Pi$, pour tous $x$ dans $E$ et $y$ dans $E_{\theta}$, on $a$ :

$$
\left(p_{\theta}(x)=y\right) \Leftrightarrow\left(\forall \chi \in X_{C} \cup\left\{\chi_{\alpha} \mid \alpha \in \theta\right\} \quad \chi(x)=\chi(y)\right) .
$$

Pour tout $\alpha$ dans $\Pi$, on note $X_{\alpha}$ la droite $V_{\alpha, \Pi}^{+}$et $V_{\alpha}^{<}$son unique supplémentaire $A$-stable. Tous les poids de $\mathbf{A}$ dans $V_{\alpha}^{<}$sont de la forme

$$
\chi_{\alpha}-\alpha-\sum_{\beta \in \Pi} n_{\beta} \beta
$$

avec, pour tout $\beta$ dans $\Pi, n_{\beta} \in \mathbb{N}$.

\subsubsection{Décomposition de Jordan}

Un élément de $G$ est dit elliptique si et seulement s'il est semi-simple et contenu dans un sous-groupe compact de $G$. Un élément de $G$ est dit hyperbolique si et seulement s'il est conjugué à un élément de $A$. On dit qu'un élément $g$ de $G$ admet une décomposition de Jordan si et seulement s'il peut s'écrire sous la forme $g=g_{e} g_{h} g_{u}$ avec $g_{e}$ elliptique, $g_{h}$ hyperbolique et $g_{u}$ unipotent qui commutent deux à deux. Dans ce cas, on note $\lambda(g)$ l'image par $\nu$ d'un élément de $A^{+}$conjugué à $g_{h}$ : il ne dépend que de $g$.

Si $\mathbb{K}$ est $\mathbb{R}$ ou $\mathbb{C}$, tous les éléments de $G$ admettent une décomposition de Jordan.

Si $\mathbb{K}$ est non-archimédien, pour tout $g$ dans $G$, il existe $n$ dans $\mathbb{N}^{*}$ tel que $g^{n}$ admette une décomposition de Jordan. On note encore $\lambda(g)=\frac{1}{n} \lambda\left(g^{n}\right)$ : il ne dépend pas de $n$.

L'application $\lambda: G \rightarrow E^{+}$est $\mathbb{R}$-analytique si $\mathbb{K}$ est $\mathbb{R}$ ou $\mathbb{C}$ et localement constante si $\mathbb{K}$ est non-archimédien. Pour tout $g$ dans $G$, on a : $\lambda\left(g^{-1}\right)=\iota(\lambda(g))$.

Soit $(\rho, V)$ une représentation rationnelle irréductible de dimension finie de $\mathbf{G}$ de plus haut poids restreint $\chi$. Pour tout $g$ dans $G$, on a $\lambda_{1}(\rho(g))=q^{\chi(\lambda(g))}$. 
Supposons $\operatorname{dim} V_{\Pi}^{+}=1$. Alors $\rho(g)$ est proximal si et seulement si, pour tout $\alpha$ dans $\Pi$ tel que $\chi-\alpha$ soit un poids de $\rho$, on a $\alpha(\lambda(g))>0$. En particulier, pour tous $\alpha$ dans $\Pi$ et $g$ dans $G, \rho_{\alpha}(g)$ est proximal dans $\mathbb{P}\left(V_{\alpha}\right)$ si et seulement si $\alpha(\lambda(g)) \neq 0$.

Soient $\theta \subset \Pi$ et $g$ dans $G$. On dit que $g$ est $\theta$-proximal si et seulement si, pour tout $\alpha$ dans $\theta, \alpha(\lambda(g))>0$, c'est-à-dire si et seulement si, pour tout $\alpha$ dans $\theta$, $\rho_{\alpha}(g)$ est proximal dans $\mathbb{P}\left(V_{\alpha}\right)$.

\subsubsection{Décomposition de Cartan}

Soit $K$ un bon sous-groupe compact maximal de $G$ relativement à $A$, c'est à dire tel que le normalisateur de $A$ dans $K$ contienne des représentants de tous les éléments de $W$.

Si $\mathbb{K}$ est $\mathbb{R}$ ou $\mathbb{C}, K$ est l'ensemble des points fixes d'une involution de Cartan $\tau$ de $G$ telle que, pour tout $a$ dans $A, \tau(a)=a^{-1}$.

On a $G=K Z^{+} K$. De plus, pour tous $z_{1}, z_{2}$ dans $Z^{+}, z_{2}$ appartient à $K z_{1} K$ si et seulement si $\nu\left(z_{1}\right)=\nu\left(z_{2}\right)$. En particulier, on a $\operatorname{ker} \nu=K \cap Z$. Il existe donc une unique application $\mu: G \rightarrow E^{+}$telle que, pour tous $g_{1}, g_{2}$ dans $G, g_{2}$ appartienne à $K g_{1} K$ si et seulement si $\mu\left(g_{1}\right)=\mu\left(g_{2}\right)$ et que $\mu_{\mid Z^{+}}=\nu_{\mid Z^{+}}$. L'application $\mu$ est propre. Elle est $\mathbb{R}$-analytique si $\mathbb{K}$ est $\mathbb{R}$ ou $\mathbb{C}$ et localement constante si $\mathbb{K}$ est non-archimédien. Pour tout $g$ dans $G$, on a $\mu\left(g^{-1}\right)=\iota(\mu(g))$ et la formule du rayon spectral :

$$
\frac{1}{n} \mu\left(g^{n}\right) \underset{n \rightarrow \infty}{\longrightarrow} \lambda(g)
$$

L'application $g \mapsto p_{\emptyset}(\mu(g))$ est un homomorphisme de $G$ dans $E^{W}$; en d'autres termes, pour tout $\chi$ dans $X_{C}$, pour tous $g, h$ dans $G$, on a $\chi(\mu(g h))=\chi(\mu(g))+$ $\chi(\mu(h))$.

Soit $(\rho, V)$ une représentation rationnelle irréductible de dimension finie de $\mathbf{G}$, de plus haut poids restreint $\chi$. Pour tout $\kappa$ dans $\mathrm{X}(\mathbf{A})$, on note $V_{\kappa}$ l'espace poids associé à $\kappa$.

Si $\mathbb{K}$ est $\mathbb{R}$ (resp. $\mathbb{C}$ ), on peut choisir un produit scalaire (resp. un produit scalaire hermitien) sur $V$ pour lequel les éléments de $\rho(K)$ sont orthogonaux (resp. unitaires) et ceux de $\rho(A)$ symétriques (resp. hermitiens). On munit $V$ de la norme

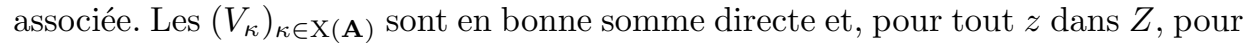
tout $\kappa$ dans $\mathrm{X}(\mathbf{A}), \rho(z)$ induit sur $V_{\kappa}$ une similitude de rapport $e^{\kappa(\nu(z))}$.

Si $\mathbb{K}$ est non-archimédien, on peut trouver, d'après $[16,6]$, une norme ultramétrique $K$-invariante sur $V$ telle que les $\left(V_{\kappa}\right)_{\kappa \in \mathrm{X}(\mathbf{A})}$ soient en bonne somme directe et que, pour tout $z$ dans $Z$, pour tout $\kappa$ dans $\mathrm{X}(\mathbf{A}), \rho(z)$ induise sur $V_{\kappa}$ une similitude de rapport $q^{\kappa(\nu(z))}$.

Dans les deux cas, on dira qu'une norme sur $V$ ayant ces propriétés est $(\rho, A, K)$ bonne. Pour une norme $(\rho, A, K)$-bonne, les éléments de $\rho(K)$ sont des isométries et ceux de $\rho(Z)$ des semi-similitudes. Pour tout $g$ dans $G$, la décomposition de Cartan permet donc d'écrire $\rho(g)$ comme le produit d'une isométrie et d'une semi- 
similitude. En particulier, on a :

$$
\|\rho(g)\|=q^{\chi(\mu(g))}
$$

et, si $k$ est un élément de $K$ tel que $g$ appartienne à $k Z K$, pour tout $v$ dans $V$ tel que $\rho(g) v$ appartienne à $k V_{\Pi}^{+}$, on a :

$$
\|\rho(g) v\|=\|\rho(g)\|\|v\| .
$$

Dorénavant, on munit, pour tout $\alpha$ dans $\Pi, V_{\alpha}$ d'une norme $\left(\rho_{\alpha}, A, K\right)$-bonne et $\mathbb{P}\left(V_{\alpha}\right)$ de la distance associée. Rappelons un résultat de $\mathrm{Y}$. Benoist :

Lemme 2.2.4. (Benoist, [3, 5.1]) Pour toute partie compacte L de G, il existe une partie compacte $M$ de $E$ telle que, pour tout $g$ dans $G$, on ait :

$$
\mu(L g L) \subset \mu(g)+M
$$

Démonstration. Soit $L$ une partie compacte de $G$. Soient $g$ dans $G$ et $l_{1}$ et $l_{2}$ dans $L$. D'une part, pour tout $\alpha$ dans $\Pi$, on a :

$$
\left\|\rho_{\alpha}\left(l_{1}\right)^{-1}\right\|^{-1}\left\|\rho_{\alpha}(g)\right\|\left\|\rho_{\alpha}\left(l_{2}\right)^{-1}\right\|^{-1} \leq\left\|\rho_{\alpha}\left(l_{1} g l_{2}\right)\right\| \leq\left\|\rho_{\alpha}\left(l_{1}\right)\right\|\left\|\rho_{\alpha}(g)\right\|\left\|\rho_{\alpha}\left(l_{2}\right)\right\|
$$

d'où, par conséquent,

$$
\chi_{\alpha}(\mu(g))-2 \max _{l \in L} \chi_{\alpha}\left(\mu\left(l^{-1}\right)\right) \leq \chi_{\alpha}\left(\mu\left(l_{1} g l_{2}\right)\right) \leq \chi_{\alpha}(\mu(g))+2 \max _{l \in L} \chi_{\alpha}(\mu(l))
$$

et, d'autre part, pour tout $\chi$ dans $X_{C}$, on a :

$$
\chi\left(\mu\left(l_{1} g l_{2}\right)\right)=\chi\left(\mu\left(l_{1}\right)\right)+\chi(\mu(g))+\chi\left(\mu\left(l_{2}\right)\right)
$$

d'où

$$
\left|\chi\left(\mu\left(l_{1} g l_{2}\right)\right)-\chi(\mu(g))\right| \leq 2 \max _{l \in L}|\chi(\mu(l))| .
$$

Le résultat en découle, puisque l'ensemble $X_{C} \cup\left\{\chi_{\alpha} \mid \alpha \in \Pi\right\}$ engendre $E^{*}$.

De même, on peut montrer :

Lemme 2.2.5. Pour tout voisinage $V$ de 0 dans $E$, il existe un voisinage $W$ de e dans $G$ tel que, pour tout $g$ dans $G$,

$$
\mu(W g W) \subset \mu(g)+V .
$$

\subsubsection{Sous-groupes paraboliques et variétés drapeaux}

Soit $\theta \subset \Pi$. On note $\mathcal{P}_{\theta}$ l'ensemble des $\mathbb{K}$-sous-groupes paraboliques conjugués à $\mathbf{P}_{\theta}$ de $\mathbf{G}$. L'application

$$
\begin{aligned}
G & \rightarrow \mathcal{P}_{\theta} \\
g & \mapsto g \mathbf{P}_{\theta} g^{-1}
\end{aligned}
$$

identifie $\mathcal{P}_{\theta}$ et $G / P_{\theta}$ : on peut ainsi voir $\mathcal{P}_{\theta}$ comme une variété $\mathbb{K}$-analytique. Comme l'action de $K$ sur $\mathcal{P}_{\theta}$ est transitive, cette variété analytique est compacte. On note $\nu_{\theta}$ l'unique probabilité borélienne $K$-invariante de $\mathcal{P}_{\theta}$. 
On note $\xi_{\theta}$ le sous-groupe $\mathbf{P}_{\theta}$ vu comme un point de $\mathcal{P}_{\theta}$ et $\mathcal{Q}_{\theta}^{-}$la sous-variété fermée $\mathcal{P}_{\theta}-P_{\theta}^{\vee} \xi_{\theta}=\mathcal{P}_{\theta}-P_{\Pi}^{\vee} \xi_{\theta}$ de $\mathcal{P}_{\theta}$.

Pour tout $\alpha$ dans $\theta, G X_{\alpha}$ est une sous-variété $\mathbb{K}$-analytique fermée de $\mathbb{P}\left(V_{\alpha}\right)$. En particulier, le $G$-entrelacement

$$
\mathcal{P}_{\theta} \rightarrow \prod_{\alpha \in \theta} \mathbb{P}\left(V_{\alpha}\right)
$$

qui, à un sous-groupe parabolique de type $\theta$, associe la famille de ses uniques points fixes dans les $V_{\alpha}, \alpha \in \theta$, est une immersion fermée. Il identifie $\xi_{\theta}$ avec $\left(X_{\alpha}\right)_{\alpha \in \theta}$ et $\mathcal{Q}_{\theta}^{-} \quad$ avec le complémentaire de l'intersection de son image et de $\prod_{\alpha \in \theta}\left(\mathbb{P}\left(V_{\alpha}\right)-\mathbb{P}\left(V_{\alpha}^{<}\right)\right)$. Pour tout $\xi$ dans $\mathcal{P}_{\theta}$, on note $\left(\xi_{\alpha}\right)_{\alpha \in \theta}$ son image par cette application. On munit $\mathcal{P}_{\theta}$ de la distance induite par la distance produit de $\prod_{\alpha \in \theta} \mathbb{P}\left(V_{\alpha}\right)$. Alors, $K$ agit par isométries et $G$ par transformations lipschitziennes sur $\mathcal{P}_{\theta}$.

Soit $g$ dans $G$. Alors $g$ est $\theta$-proximal si et seulement s'il possède un point fixe attracteur dans $\mathcal{P}_{\theta}$. On note alors $\xi_{\theta, g}^{+}$ce point fixe : il s'identifie à $\left(V_{\alpha, \rho_{\alpha}(g)}^{+}\right)_{\alpha \in \theta}$.

Soit $L \subset P_{\Pi}$ une partie bornée. L'ensemble $\bigcup_{z \in Z^{+}} z^{-1} L z$ est encore borné. De même, si $L \subset P_{\Pi}^{\vee}$ est une partie bornée, l'ensemble $\bigcup_{z \in Z^{+}} z L z^{-1}$ est encore borné. Pour tout $\varepsilon>0$, on pose :

$$
\left.B_{\theta}^{\varepsilon}=\left\{\xi \in \mathcal{P}_{\theta} \mid \forall \alpha \in \theta \quad d\left(\xi_{\alpha}, \mathbb{P}\left(V_{\alpha}^{<}\right)\right) \geq \varepsilon\right)\right\} .
$$

Il existe une partie compacte $L$ de $P_{\Pi}^{\vee}$ telle que $B_{\theta}^{\varepsilon} \subset L \xi_{\theta}$.

\subsubsection{Sous-groupes Zariski denses}

Nous rappelons ici une partie des résultats de [4, 4] et de [5].

Soit $\Gamma$ un sous-groupe Zariski dense de $G$. On appelle cône limite de $\Gamma$ et on note $l_{\Gamma}$ le cône fermé engendré par $\lambda(\Gamma)$ dans $E^{+}$.

Soit $P \subset E$. On appelle cône asymptote à $P$ l'ensemble des vecteurs $x$ dans $E$ pour lesquels il existe une suite de vecteurs $\left(x_{n}\right)_{n \in \mathbb{N}}$ dans $P$ et une suite de réels positifs $\left(t_{n}\right)_{n \in \mathbb{N}}$ tendant vers 0 telles que $t_{n} x_{n} \underset{n \rightarrow \infty}{\longrightarrow} x$.

Par la formule du rayon spectral, $l_{\Gamma}$ est contenu dans le cône asymptote à $\mu(\Gamma)$.

Théorème 2.2.6. (Benoist, [4]) Le cône limite $l_{\Gamma}$ de $\Gamma$ est exactement le cône asymptote à $\mu(\Gamma)$ et l'ensemble $\mu(\Gamma)$ reste à distance bornée de $l_{\Gamma}$. Le cône $l_{\Gamma}$ est convexe et, si $\mathbb{K}$ est $\mathbb{R}$, son intersection avec $E_{S}$ est d'intérieur non vide dans $E_{S}$.

On appelle type de $\Gamma$ et on note $\theta_{\Gamma}$ l'unique partie $\theta$ de $\Pi$ telle que $l_{\Gamma} \subset E_{\theta}^{+}$ et que $l_{\Gamma} \cap E_{\theta}^{++} \neq \emptyset$ : c'est le plus grand $\theta \subset \Pi$ tel que $\Gamma$ contienne des éléments $\theta$-proximaux. Si $\mathbb{K}$ est $\mathbb{R}, \theta_{\Gamma}=\Pi$. L'ensemble $\theta_{\Gamma}$ est stable par $\iota$ et, si $\Gamma$ est discret, $\theta_{\Gamma} \neq \emptyset$.

On note $F_{\Gamma}$ le sous-espace vectoriel de $E$ engendré par $l_{\Gamma}$. Nous aurons à utiliser l'existence dans $\Gamma$ de sous-semi-groupes libres : 
Proposition 2.2.7. (Benoist, $[4,5.1])$ Si $\theta_{\Gamma} \neq \emptyset$, il existe un réel $\kappa \geq 0$ tel que, pour tout cône $\mathcal{C}$ dans $E$, si l'intérieur dans $F_{\Gamma}$ de $\mathcal{C} \cap l_{\Gamma}$ est non vide, il existe des éléments $\gamma_{1}$ et $\gamma_{2}$ dans $\Gamma$ et des vecteurs $x_{1}$ et $x_{2}$ dans $\mathcal{C} \cap l_{\Gamma}$ tels que le sous-semi-groupe $\Delta$ de $\Gamma$ engendré par $\gamma_{1}$ et $\gamma_{2}$ soit libre et que, si $\Phi$ est l'unique homomorphisme de semi-groupes envoyant $\gamma_{1}$ sur $x_{1}$ et $\gamma_{2}$ sur $x_{2}$, pour tout $\gamma$ dans $\Delta$, mot de longueur $l$ en les générateurs $\gamma_{1}$ et $\gamma_{2}$, on ait :

$$
\mu(\gamma) \in \mathcal{C} \text { et }\|\mu(\gamma)-\Phi(\gamma)\| \leq \kappa l
$$

\subsection{Produit générique}

Dans cette section nous allons démontrer :

Proposition 2.3.1. Soit $\Gamma$ un sous-groupe discret Zariski dense de $G$. Il existe une application $\pi: \Gamma \times \Gamma \rightarrow \Gamma$ ayant les propriétés suivantes :

(i) il existe un réel $\kappa \geq 0$ tel que, pour tous $\gamma_{1}, \gamma_{2}$ dans $\Gamma$,

$$
\left\|\mu\left(\pi\left(\gamma_{1}, \gamma_{2}\right)\right)-\mu\left(\gamma_{1}\right)-\mu\left(\gamma_{2}\right)\right\| \leq \kappa .
$$

(ii) pour tout réel $R \geq 0$, il existe une partie finie $H$ de $\Gamma$ telle que, pour $\gamma_{1}, \gamma_{2}, \gamma_{1}^{\prime}, \gamma_{2}^{\prime}$ dans $\Gamma$, avec $\left\|\mu\left(\gamma_{1}\right)-\mu\left(\gamma_{1}^{\prime}\right)\right\| \leq R$ et $\left\|\mu\left(\gamma_{2}\right)-\mu\left(\gamma_{2}^{\prime}\right)\right\| \leq R$,

$$
\pi\left(\gamma_{1}, \gamma_{2}\right)=\pi\left(\gamma_{1}^{\prime}, \gamma_{2}^{\prime}\right) \Rightarrow\left(\gamma_{1}^{\prime} \in \gamma_{1} H \text { et } \gamma_{2}^{\prime} \in H \gamma_{2}\right) .
$$

L'idée de la construction est d'écrire, pour $\gamma_{1}, \gamma_{2}$ dans $\Gamma, \pi\left(\gamma_{1}, \gamma_{2}\right)=\gamma_{1} f_{\gamma_{1}, \gamma_{2}} \gamma_{2}$ où $f_{\gamma_{1}, \gamma_{2}}$ est choisi dans une partie finie de $\Gamma$ de façon à vérifier les hypothèses des lemmes 2.3.3 et 2.3.8.

Dorénavant, on fixe, pour tout élément $g$ de $G$, un élément $z_{g}$ de $Z^{+}$et des éléments $k_{g}$ et $l_{g}$ de $K$ tels que $g=k_{g} z_{g} l_{g}$.

\subsubsection{Un calcul de composante de Cartan}

Nous effectuons ici le calcul qui permet de valider le point (i) de la proposition 2.3.1.

Soit $(\rho, V)$ une représentation rationnelle irréductible de dimension finie de G munie d'une norme $(\rho, A, K)$-bonne. On munit $\mathbb{P}(V)$ de la distance associée. Rappelons que, pour tout $z$ dans $Z^{+}$, si, pour tout $\alpha$ dans $\Pi, \alpha(\nu(z))>0, \rho(z)$ est une semi-similitude avec $V_{\rho(z)}^{M}=V_{\Pi}^{+}$et $V_{\rho(z)}^{m}=V_{\Pi}^{<}$et que, pour tout $g$ dans $G$, pour tout $v$ dans $V$ tel que $\rho(g) v$ appartienne à $k_{g} V_{\Pi}^{+}$, on a :

$$
\|\rho(g) v\|=\|\rho(g)\|\|v\| .
$$

Pour tout $g$ dans $G$, on note :

$$
V_{\rho, g}^{M}=k_{g} V_{\Pi}^{+} \text {et } V_{\rho, g}^{m}=l_{g}^{-1} V_{\Pi}^{<} .
$$

Pour $\alpha$ dans $\Pi$, on notera $V_{\alpha, g}^{M}$ et $V_{\alpha, g}^{m}$ pour $V_{\rho_{\alpha}, g}^{M}$ et $V_{\rho_{\alpha}, g}^{m}$. 
Si $\mathbb{K}$ est $\mathbb{R}$ ou $\mathbb{C}$ et si, pour tout $\alpha$ dans $\Pi, \alpha(\mu(g))>0, V_{\rho, g}^{M}$ et $V_{\rho, g}^{m}$ ne dépendent pas des $k_{g}$ et $l_{g}$ choisis.

En appliquant le lemme 2.1.4 aux représentations de $\mathbf{G}$, on obtient :

Lemme 2.3.2. Soit $(\rho, V)$ une représentation rationnelle irréductible de dimension finie de $\mathbf{G}$ munie d'une norme $(\rho, A, K)$-bonne. Pour tous $\varepsilon>0$ et $g$ dans $G$, on $a$ :

$$
\forall v \in V-\{0\} \quad\left(d\left(\mathbb{K} v, \mathbb{P}\left(V_{\rho, g}^{m}\right)\right) \geq \varepsilon\right) \Rightarrow(\|g v\| \geq \varepsilon\|\rho(g)\|\|v\|) .
$$

Démonstration. Comme $K$ agit par isométries sur $V$, il suffit de démontrer ce résultat quand $g$ est dans $Z^{+}$. Alors, $\rho(g)$ est une semi-similitude et $V_{\rho(g)}^{m}$ est contenu dans $V_{\Pi}^{<}$. Le résultat est alors une conséquence du lemme 2.1.4.

Nous sommes à présent en mesure d'effectuer le calcul de la composante de Cartan du produit générique. L'hypothèse sur les distances dans l'énoncé ci-dessous traduit le fait que $f$ écarte suffisament les uns des autres les ensembles de drapeaux associés à $g$ et à $h$.

Lemme 2.3.3. Soient $\theta \subset \Pi, r>0$ et $F$ une partie compacte de $G$. Il existe un réel $\kappa \geq 0$ tel que, pour tous $g, h$ dans $G$, pour tout $f$ dans $F$,

$$
\left(\forall \alpha \in \theta \quad d\left(f V_{\alpha, h}^{M}, \mathbb{P}\left(V_{\alpha, g}^{m}\right)\right) \geq r\right) \Rightarrow\left(\left\|p_{\theta}(\mu(g f h)-\mu(g)-\mu(h))\right\| \leq \kappa\right) .
$$

Démonstration. Comme on l'a vu au paragraphe 2.2.5., pour tous $g$ et $h$ dans $G$, pour tout $f$ dans $F$, pour tout $\chi$ dans $X_{C}$, on a :

$$
\chi(\mu(g f h))=\chi(\mu(g))+\chi(\mu(f))+\chi(\mu(h))
$$

et, donc,

$$
|\chi(\mu(g f h)-\mu(g)-\mu(h))| \leq \max _{f \in F}|\chi(\mu(f))| .
$$

Par ailleurs, d'après le lemme 2.3.2, pour tout $g$ dans $G$, pour tout $\alpha$ dans $\theta$, on a :

$$
\forall v \in V_{\alpha} \quad\left(d\left(\mathbb{K} v, \mathbb{P}\left(V_{\alpha, g}^{m}\right)\right) \geq r\right) \Rightarrow\left(\|g v\| \geq r\left\|\rho_{\alpha}(g)\right\|\|v\|\right) .
$$

Soient $g$ et $h$ dans $G$. Soit $f$ dans $F$ tels que, pour tout $\alpha$ dans $\theta$, on ait :

$$
d\left(f V_{\alpha, h}^{M}, \mathbb{P}\left(V_{\alpha, g}^{m}\right)\right) \geq r .
$$

Soit $\alpha$ dans $\theta$. D'une part, on a :

$$
\begin{aligned}
\left\|\rho_{\alpha}(g f h)\right\| & \leq\left\|\rho_{\alpha}(g)\right\|\left\|\rho_{\alpha}(h)\right\|\left\|\rho_{\alpha}(f)\right\| \\
& \leq\left\|\rho_{\alpha}(g)\right\|\left\|\rho_{\alpha}(h)\right\| \max _{k \in F}\left\|\rho_{\alpha}(k)\right\|
\end{aligned}
$$

donc,

$$
\chi_{\alpha}(\mu(g f h)-\mu(g)-\mu(h)) \leq \max _{k \in F} \chi_{\alpha}(\mu(k)) .
$$


D'autre part, soit $v$ un vecteur non nul de $V_{\alpha}$ tel que $h v \in V_{\alpha, h}^{M}$. On a :

$$
\begin{aligned}
\left\|\rho_{\alpha}(g f h) v\right\| & \geq r\left\|\rho_{\alpha}(g)\right\|\left\|\rho_{\alpha}(f h) v\right\| \\
& \geq r\left\|\rho_{\alpha}(g)\right\|\left\|\rho_{\alpha}(f)^{-1}\right\|^{-1}\left\|\rho_{\alpha}(h) v\right\| \\
& \geq r\left\|\rho_{\alpha}(g)\right\|\left\|\rho_{\alpha}(f)^{-1}\right\|^{-1}\left\|\rho_{\alpha}(h)\right\|\|v\| .
\end{aligned}
$$

Il vient :

$$
\left\|\rho_{\alpha}(g f h)\right\| \geq r\left(\max _{k \in F}\left\|\rho_{\alpha}(k)^{-1}\right\|\right)^{-1}\left\|\rho_{\alpha}(g)\right\|\left\|\rho_{\alpha}(h)\right\|
$$

d'où

$$
\chi_{\alpha}(\mu(g f h)-\mu(g)-\mu(h)) \geq \log _{q} r-\max _{k \in F} \chi_{\alpha}\left(\mu\left(k^{-1}\right)\right) .
$$

Le résultat en découle, puisque, d'après le lemme 2.2.3, pour tout $x$ dans $E, p_{\theta}(x)$ est entièrement déterminé par les $\chi(x)$, pour $\chi$ dans $X_{C} \cup\left\{\chi_{\alpha} \mid \alpha \in \theta\right\}$.

\subsubsection{Un résultat de finitude}

Rappelons que, si $V$ est un $\mathbb{K}$-espace vectoriel, une famille de droites $\left(X_{j}\right)_{j \in J}$ est dite être en position générale si et seulement si pour toute partie finie $K$ de $J$ de cardinal $\leq \operatorname{dim} V$, la famille de droites $\left(X_{j}\right)_{j \in K}$ est en somme directe. Si $J$ est fini, l'ensemble des familles de droites en position générale est un ouvert de Zariski de $\mathbb{P}(V)^{J}$.

Le résultat suivant est dû à H. Abels, G.-A. Margulis et G.-A. Soifer ([2, 4.7]). Il nous permettra de trouver, dans un sous-groupe Zariski dense $\Gamma$ de $G$, une partie finie $F$ telle que, étant donnés deux éléments $g$ et $h$ de $G$, il existe $f$ dans $F$ vérifiant les hypothèses des lemmes 2.3 .3 et 2.3 .8 .

Si $(\rho, V)$ est une représentation rationnelle irréductible et de dimension finie de $\mathbf{G}$, de plus haut poids restreint $\chi$, on note $\theta_{\rho}$ l'ensemble des $\alpha$ dans $\Pi$ tels que $\chi-\alpha$ soit un poids de $\rho$.

Proposition 2.3.4. (Abels-Margulis-Soifer) Soit $\Gamma$ un sous-groupe Zariski dense de $G$. Soit $\left(\rho_{i}, V_{i}\right)_{i \in I}$ une famille finie de représentations rationnelles, irréductibles et de dimensions finies de $\mathbf{G}$, chacune munie d'une norme. On suppose que, pour tout $i$ dans $I$, on a $\theta_{\rho_{i}} \subset \theta_{\Gamma}$. Alors, il existe une partie finie $F$ de $\Gamma$ et un réel $r>0$ ayant la propriété suivante : pour toutes familles $\left(X_{i}\right)_{i \in I}$ et $\left(Y_{i}\right)_{i \in I}$ où, pour tout $i$ dans $I, X_{i}$ est une droite et $Y_{i}$ un hyperplan de $V_{i}$, il existe $f$ dans $F$ tel que, pour tout $i$ dans $I$,

$$
d\left(f X_{i}, \mathbb{P}\left(Y_{i}\right)\right) \geq r .
$$

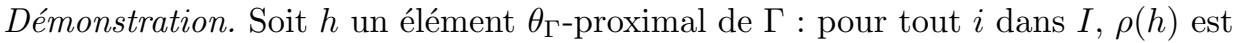
proximal dans $\mathbb{P}\left(V_{i}\right)$. Notons, pour simplifier,

$$
V_{i}^{+}=V_{i, \rho_{i}(h)}^{+} \text {et } V_{i}^{<}=V_{i, \rho_{i}(h)}^{<} .
$$


Soit $l \in \mathbb{N}$. Par récurrence, comme $\Gamma$ est Zariski dense dans $G$, qui est Zariski connexe, et comme les $\left(\rho_{i}, V_{i}\right)_{i \in I}$ sont irréductibles, on peut construire une famille $\left(g_{j}\right)_{1 \leq j \leq l}$ d'éléments de $\Gamma$ telle que, pour tout $i$ dans $I$,

(i) la famille de droites $\left(g_{j} V_{i}^{+}\right)_{1 \leq j \leq l}$ est en position générale.

(ii) la famille d'hyperplans $\left(g_{i} V_{i}^{<}\right)_{1 \leq i \leq l}$ est en position générale.

(iii) pour tous $j, k$ dans $\llbracket 1, l \rrbracket, g_{j} V_{i}^{+} \not \subset g_{k} V_{i}^{<}$.

Supposons $l \geq \sum_{i \in I} \operatorname{dim} V_{i}$. Alors, on peut trouver $r>0$ tel que, pour toutes familles $\left(X_{i}\right)_{i \in I}$ et $\left(Y_{i}\right)_{i \in I}$ où, pour tout $i$ dans $I, X_{i}$ est une droite et $Y_{i}$ un hyperplan de $V_{i}$,

(i) il existe $j$ dans $\llbracket 1, l \rrbracket$ tel que, pour tout $i$ dans $I$,

$$
d\left(X_{i}, \mathbb{P}\left(g_{j} V_{i}^{<}\right)\right) \geq r .
$$

(ii) il existe $k$ dans $\llbracket 1, l \rrbracket$ tel que, pour tout $i$ dans $I$,

$$
d\left(g_{k} V_{i}^{+}, \mathbb{P}\left(Y_{i}\right)\right) \geq r .
$$

(iii) pour tous $j, k$ dans $\llbracket 1, l \rrbracket$,

$$
d\left(g_{j} V_{i}^{+}, \mathbb{P}\left(g_{k} V_{i}^{<}\right)\right) \geq r .
$$

Pour tout $j$ dans $\llbracket 1, l \rrbracket$, on pose $V_{i, j}^{+}=g_{j} V_{i}^{+}$et $V_{i, j}^{<}=g_{j} V_{i}^{<}$.

Choisissons, pour tout $j$ dans $\llbracket 1, l \rrbracket$, un entier $n_{j}$ suffisament grand pour que, pour tout $i$ dans $I, \rho\left(g_{j} h^{n_{j}} g_{j}^{-1}\right)$ envoie

$$
B\left(\mathbb{P}\left(V_{i, j}^{<}\right), \frac{r}{2}\right) \text { dans } b\left(V_{i, j}^{+}, \frac{r}{2}\right)
$$

et posons, pour tout $j$ dans $\llbracket 1, l \rrbracket, h_{j}=g_{j} h^{n_{j}} g_{j}^{-1}$.

Posons $F=\left\{h_{k} h_{j} \mid 1 \leq j, k \leq l\right\}$ et montrons que la partie $F$ et le réel $\frac{r}{2}$ vérifient les conclusions de la proposition.

Soient, pour tout $i$ dans $I, X_{i}$ une droite de $V_{i}$ et $Y_{i}$ un hyperplan de $V_{i}$. Il existe $j$ dans $\llbracket 1, l \rrbracket$ tel que, pour tout $i$ dans $I$,

$$
d\left(X_{i}, \mathbb{P}\left(V_{i, j}^{<}\right)\right) \geq r
$$

et, par conséquent,

$$
d\left(h_{j} X_{i}, V_{i, j}^{+}\right) \leq \frac{r}{2} .
$$

Par ailleurs, il existe $k$ dans $\llbracket 1, l \rrbracket$ tel que, pour tout $i$ dans $I$,

$$
d\left(V_{i, k}^{+}, \mathbb{P}\left(Y_{i}\right)\right) \geq r .
$$

Or, pour tout $i$ dans $I$, comme

on a

$$
d\left(V_{i, j}^{+}, \mathbb{P}\left(V_{i, k}^{<}\right)\right) \geq r
$$

$$
d\left(h_{j} X_{i}, \mathbb{P}\left(V_{i, k}^{<}\right)\right) \geq \frac{r}{2}
$$


et, donc,

$$
d\left(h_{k} h_{j} X_{i}, \mathbb{P}\left(V_{i, k}^{+}\right)\right) \leq \frac{r}{2} .
$$

Il vient :

$$
d\left(h_{k} h_{j} X_{i}, \mathbb{P}\left(Y_{i}\right)\right) \geq \frac{r}{2},
$$

ce qu'il fallait démontrer.

Déduisons-en un résultat qui sera utilisé dans la démonstration du lemme 2.3.8:

Corollaire 2.3.5. Il existe $\varepsilon>0$ tel que, pour toutes familles $\left(U_{\alpha}\right)_{\alpha \in \Pi}$ et $\left(W_{\alpha}\right)_{\alpha \in \Pi}$, où, pour tout $\alpha$ dans $\Pi, U_{\alpha}$ et $W_{\alpha}$ sont des hyperplans de $V_{\alpha}$, il existe $\xi$ dans $\mathcal{P}_{\Pi}$ avec, pour tout $\alpha$ dans $\Pi$,

$$
d\left(\xi_{\alpha}, \mathbb{P}\left(U_{\alpha}\right)\right) \geq \varepsilon \text { et } d\left(\xi_{\alpha}, \mathbb{P}\left(W_{\alpha}\right)\right) \geq \varepsilon .
$$

Démonstration. Soit $r>0$ comme dans la proposition 2.3 .4 avec $\Gamma=G$ et, comme famille de représentations, la réunion de deux copies de $\left(V_{\alpha}\right)_{\alpha \in \Pi \text {. Soient, pour tout }}$ $\alpha$ dans $\Pi, U_{\alpha}$ et $W_{\alpha}$ des hyperplans de $V_{\alpha}$. Alors, il existe $f$ dans $G$ tel que, pour tout $\alpha$ dans $\Pi$, on ait :

$$
d\left(f X_{\alpha}, \mathbb{P}\left(U_{\alpha}\right)\right) \geq r \text { et } d\left(f X_{\alpha}, \mathbb{P}\left(W_{\alpha}\right)\right) \geq r .
$$

Le point $\xi=f \xi_{\Pi}$ convient.

\subsubsection{Un contrôle de distance}

Ce paragraphe et le suivant ont pour but d'établir les résultats intermédiaires permettant de démontrer le point (ii) de la proposition 2.3.1. Nous commençons ici par généraliser des phénomènes de géométrie à courbure strictement négative.

Soit toujours $\theta \subset \Pi$. On note $K_{\theta}$ le groupe $P_{\theta} \cap K$ et, pour tout $C \geq 0$, on pose

$$
E_{\theta}^{C}=\left\{x \in E^{+} \mid \forall \alpha \in \theta^{c} \quad \alpha(x) \leq C\right\}
$$

et $Z_{\theta}^{C}=\nu^{-1}\left(E_{\theta}^{C}\right)$.

Dans $[1,3.5], P$. Albuquerque démontrait une généralisation du lemme des ombres de Sullivan. Le lemme suivant est la contraposée de ce résultat : à partir d'une information sur les actions d'un élément $k$ de $K$ et d'un élément $z$ de $Z$ sur une variété drapeau, il permet de contrôler la distance entre $z$ et $k z$.

Lemme 2.3.6. Pour tout $C \geq 0$ et pour toute partie compacte $L$ de $P_{\Pi}^{\vee}$, il existe une partie compacte $M$ de $G$ telle que, pour tout $z$ dans $Z_{\theta}^{C}$, pour tout $k$ dans $K$,

$$
\left(k \xi_{\theta} \in z L \xi_{\theta}\right) \Rightarrow\left(z^{-1} k z \in M\right) .
$$

Démonstration. Donnons-nous $C$ et $L$ comme dans l'énoncé. On peut supposer que, pour tout $z$ dans $Z^{+}, z L z^{-1} \subset L$. 
Pour tout $z$ dans $Z^{+}$et pour tout $p$ dans $L$, choisissons $k(z, p)$ dans $K$ et $q(z, p)$ dans $P_{\Pi}$ tels que $z p=k(z, p) q(z, p)$, i.e. $k(z, p) q(z, p)$ est une décomposition d'Iwasawa de $z p$.

Pour $z$ dans $Z^{+}$et $p$ dans $L$, on a $: q(z, p) z^{-1}=k(z, p)^{-1} z p z^{-1} \in K L$ et, donc, la partie de $P_{\Pi}$

$$
L^{\prime}=\left\{q(z, p) z^{-1} \mid z \in Z^{+}, p \in L\right\}
$$

est bornée. Par conséquent,

$$
L^{\prime \prime}=\left\{z^{-1} q(z, p) \mid z \in Z^{+}, p \in L\right\} \subset \bigcup_{z \in Z^{+}} z^{-1} L^{\prime} z
$$

est bornée. Or, pour tout $z$ dans $Z^{+}$, pour tout $p$ dans $L$,

$$
z^{-1} k(z, p) z=z^{-1}\left(z p q(z, p)^{-1}\right) z=p\left(z^{-1} q(z, p)\right)^{-1} \in L\left(L^{\prime \prime}\right)^{-1} .
$$

Par ailleurs, l'ensemble

$$
L^{\prime \prime \prime}=\bigcup_{z \in Z_{\theta}^{C}} z^{-1} K_{\theta} z
$$

est borné.

Soient alors $k$ dans $K$ et $z$ dans $Z_{\theta}^{C}$ tels que $k \xi_{\theta} \in z L \xi_{\theta}$. Écrivons $k \xi_{\theta}=z p \xi_{\theta}$ avec $p$ dans $L$. On a :

$$
k \xi_{\theta}=k(z, p) \xi_{\theta}
$$

i.e. $k \in k(z, p) K_{\theta}$ et, donc,

$$
z^{-1} k z \in\left(z^{-1} k(z, p) z\right)\left(z^{-1} K_{\theta} z\right) \subset L\left(L^{\prime \prime}\right)^{-1} L^{\prime \prime \prime} .
$$

Pour tous $g$ dans $G$ et $\varepsilon>0$, on note

$$
B_{\theta, g}^{\varepsilon}=l_{g}^{-1} B_{\theta}^{\varepsilon} \text {. }
$$

Nous faisons jouer à l'ensemble $g B_{\theta, g}^{\varepsilon}$ le rôle des ombres de [18] et [1].

L'énoncé du résultat suivant signifie que, si un élément de $G$ a une composante de Cartan proche de la facette associé à $\theta$, on peut reconstituer cet élément, à un compact près, à partir de sa composante de Cartan et de son ombre dans $\mathcal{P}_{\theta}$.

Proposition 2.3.7. Pour tous $C \geq 0$ et $\varepsilon>0$, il existe une partie compacte $M$ de $G$ telle que, pour tout $\theta \subset \Pi$, pour tous $g, h$ dans $G$ avec $\mu(g) \in E_{\theta}^{C}$ et $\mu(h) \geq_{C} \mu(g)$, si $g B_{\theta, g}^{\varepsilon} \cap h B_{\theta, h}^{\varepsilon} \neq \emptyset$, alors on $a$ :

$$
g \in k_{h} z_{g} M
$$

Démonstration. Choisissons une partie compacte $L$ de $P_{\Pi}^{\vee}$ telle que $B_{\theta}^{\varepsilon} \subset L \xi_{\theta}$ et que, pour tout $z$ dans $Z^{+}, z L z^{-1} \subset L$. Posons :

$$
L^{\prime}=\bigcup_{\substack{z \in Z \\ \forall \alpha \in \Pi|\alpha(\nu(z))| \leq C}} z^{-1} L z .
$$


L'ensemble $L^{\prime}$ est encore borné.

D'après le lemme 2.3.6, on peut trouver une partie compacte $M$ de $G$ telle que, pour tout $z$ dans $Z_{\theta}^{C}$, pour tout $k$ dans $K$,

$$
\left(k \xi_{\theta} \in z L^{\prime} \xi_{\theta}\right) \Rightarrow(k z \in z M) .
$$

Par ailleurs, on peut trouver une partie compacte $M^{\prime}$ de $G$ telle que, pour tout $z$ dans $Z_{\theta}^{C}, z^{-1} K_{\theta} z \subset M^{\prime}$.

Soient $g$ et $h$ comme dans l'énoncé. On a :

$$
g B_{\theta, g}^{\varepsilon} \subset k_{g} z_{g} L \xi_{\theta} \text { et } h B_{\theta, h}^{\varepsilon} \subset k_{h} z_{h} L \xi_{\theta} .
$$

Comme $\mu(h)=\nu\left(z_{h}\right) \geq_{C} \mu(g)=\nu\left(z_{g}\right)$, il vient :

$$
k_{h} z_{h} L \xi_{\theta} \subset k_{h} z_{g} L^{\prime} \xi_{\theta}
$$

et, donc,

$$
k_{g} z_{g} L^{\prime} \xi_{\theta} \cap k_{h} z_{g} L^{\prime} \xi_{\theta} \neq \emptyset .
$$

Soit $\xi \in k_{g} z_{g} L^{\prime} \xi_{\theta} \cap k_{h} z_{g} L^{\prime} \xi_{\theta}$. Soit $m_{1}$ dans $K$ tel que $\xi=k_{g} m_{1} \xi_{\theta}$.

On a :

$$
m_{1} \xi_{\theta} \in z_{g} L^{\prime} \xi_{\theta}
$$

et, donc,

$$
m_{1} z_{g} \in z_{g} M \text { ou encore } k_{g} m_{1} z_{g} \in g K M \text {. }
$$

Soient $h^{\prime}=k_{h} z_{g} l_{h}$ et $m_{2}$ dans $K$ tels que $\xi=k_{g} m_{2} \xi_{\theta}$. On a, de même,

$$
k_{h} m_{2} z_{g} \in h^{\prime} K M \text {. }
$$

Or, comme

$$
k_{g} m_{1} \xi_{\theta}=\xi=k_{h} m_{2} \xi_{\theta},
$$

on a :

$$
k_{g} m_{1} \in k_{h} m_{2} K_{\theta} .
$$

Il vient :

$$
g \in k_{g} m_{1} z_{g} M^{-1} K \subset k_{h} m_{2} K_{\theta} z_{g} M^{-1} K \subset h^{\prime} K M M^{\prime} M^{-1} K .
$$

\subsubsection{Produit générique dans $G$}

Le résultat suivant combine la proposition 2.3 .7 et les raisonnements de la section 2.1.

Lemme 2.3.8. Soient $\theta \subset \Pi, C>0, r>0$ et $F$ une partie compacte de $G$. Il existe une partie compacte $M$ de $G$ ayant la propriété suivante : soient $g, h$ dans $G$ et $f$ dans $F$; si

$$
\mu(g), \mu(g f h) \in E_{\theta}^{C} \text { et si } \forall \alpha \in \theta \quad d\left(f V_{\alpha, h}^{M}, \mathbb{P}\left(V_{\alpha, g}^{m}\right)\right) \geq r,
$$

alors on $a$ :

$$
g \in k_{g f h} z_{g} M .
$$


Ce lemme signifie que, si $f, g$ et $h$ sont des éléments de $G$ et que $f$ met les ensembles de drapeaux associés à $g$ et $h$ en position suffisament générale, on peut retrouver $g$, à un compact près, à partir de sa composante de Cartan et de $g f h$. Comme on sait, d'après le lemme 2.3.3, que, quitte à augmenter $C$, sous nos hypothèses, on a $\mu(g f h) \geq_{C} \mu(g)$, on va chercher à appliquer la proposition 2.3.7, et, donc, à montrer que, pour un $\varepsilon>0$, on a $g B_{\theta, g}^{\varepsilon} \cap(g f h) B_{\theta, g f h}^{\varepsilon} \neq \emptyset$, ou encore $B_{\theta, g}^{\varepsilon} \cap f h B_{\theta, g f h}^{\varepsilon} \neq \emptyset$. Pour montrer cette dernière propriété, on appliquera le lemme suivant à $h$ :

Lemme 2.3.9. Soit $(\rho, V)$ une représentation rationnelle irréductible de dimension finie de $\mathbf{G}$, munie d'une norme $(\rho, A, K)$-bonne. Pour tous $r>0$ et $\varepsilon>0$, il existe un réel $\eta>0$ tels que, pour tout $g$ dans $G$, pour tout hyperplan $W$ de $V$, si $\delta\left(V_{\rho, g}^{M}, \mathbb{P}(W)\right) \geq r$, on $a:$

$$
g^{-1} b(\mathbb{P}(W), \eta) \subset b\left(\mathbb{P}\left(g^{-1} W\right), \varepsilon\right) .
$$

Démonstration. Comme $K$ agit par isométries sur $\mathbb{P}(V)$, il suffit de le démontrer pour $g$ dans $Z^{+}$. Alors, $\rho(g)$ est une semi-similitude et notre résultat est le lemme 2.1.6.

Démonstration du lemme 2.3.8. Comme $G$ agit par transformations lipschitziennes sur les $\mathbb{P}\left(V_{\alpha}\right), \alpha \in \Pi$, on peut trouver $r^{\prime}>0$ tel que, pour tout $f$ dans $F$, pour tout $\alpha$ dans $\theta$ et pour tous $X, Y$ dans $\mathbb{P}\left(V_{\alpha}\right)$, on ait

$$
(d(f X, Y) \geq r) \Rightarrow\left(d\left(X, f^{-1} Y\right) \geq r^{\prime}\right) .
$$

Soit $\varepsilon>0$ comme dans le corollaire 2.3.5.

D'après le lemme 2.3.9, il existe un réel $0<\eta \leq \varepsilon$ vérifiant la propriété suivante : soient $g$ dans $G$ et, pour tout $\alpha$ dans $\theta$, un hyperplan $W_{\alpha}$ de $V_{\alpha}$ avec

$$
d\left(\mathbb{P}\left(V_{\alpha, g}^{M}\right), \mathbb{P}\left(W_{\alpha}\right)\right) \geq r^{\prime},
$$

alors, on a

$$
\left.\forall \alpha \in \theta \quad g^{-1} b\left(\mathbb{P}\left(W_{\alpha}\right)\right), \eta\right) \subset b\left(\mathbb{P}\left(g^{-1} W_{\alpha}\right), \varepsilon\right) .
$$

Il existe un réel $0<\varpi \leq \eta$ tel que, pour tout $\alpha$ dans $\theta$, pour tout hyperplan $W$ de $V_{\alpha}$, pour tout $f$ dans $F$, on ait :

$$
f^{-1} b(\mathbb{P}(W), \varpi) \subset b\left(f^{-1} \mathbb{P}(W), \eta\right) .
$$

Par ailleurs, d'après le lemme 2.3.3, quitte à augmenter $C$, on peut supposer que, pour tous $g, h$ dans $G$, pour tout $f$ dans $F$, si

$$
\forall \alpha \in \theta \quad d\left(f V_{\alpha, h}^{M}, \mathbb{P}\left(V_{\alpha, g}^{m}\right)\right) \geq r,
$$

on a $p_{\theta}(\mu(g f h)) \geq_{C} p_{\theta}(\mu(g))$. Alors, si $\mu(g), \mu(g f h)$ sont dans $E_{\theta}^{C}$, on a $\mu(g f h) \geq_{3 C}$ $\mu(g)$.

Enfin, d'après la proposition 2.3.7, il existe une partie compacte $M$ de $G$ telle que, pour tout $\theta \subset \Pi$, pour tous $g, h$ dans $G$ avec $\mu(g) \in E_{\theta}^{C}$ et $\mu(h) \geq_{3 C} \mu(g)$, si 
$g B_{\theta, g}^{\varepsilon} \cap h B_{\theta, h}^{\varepsilon} \neq \emptyset$, alors on a :

$$
g \in k_{h} z_{g} M
$$

Soient $g, h$ dans $G$ et $f$ dans $F$ avec $\mu(g), \mu(g f h) \in E_{\theta}^{C}$. Supposons que l'on a :

$$
\forall \alpha \in \theta \quad d\left(f V_{\alpha, h}^{M}, \mathbb{P}\left(V_{\alpha, g}^{m}\right)\right) \geq r,
$$

On a $\mu(g f h) \geq_{3 C} \mu(g)$. Par ailleurs, pour tout $\alpha$ dans $\theta$, on a :

$$
h^{-1} b\left(f^{-1} \mathbb{P}\left(V_{\alpha, g}^{m}\right), \eta\right) \subset b\left(h^{-1} f^{-1} \mathbb{P}\left(V_{\alpha, g}^{m}\right), \varepsilon\right)
$$

ce qui implique :

$$
h^{-1} f^{-1} b\left(\mathbb{P}\left(V_{\alpha, g}^{m}\right), \varpi\right) \subset b\left(h^{-1} f^{-1} \mathbb{P}\left(V_{\alpha, g}^{m}\right), \varepsilon\right) .
$$

On peut trouver $\xi$ dans $\mathcal{P}_{\theta}$ tel que, pour tout $\alpha$ dans $\theta$, on ait :

$$
d\left(\xi_{\alpha}, \mathbb{P}\left(V_{\alpha, g f h}^{m}\right)\right) \geq \varepsilon \text { et } d\left(\xi_{\alpha}, h^{-1} f^{-1} \mathbb{P}\left(V_{\alpha, g}^{m}\right)\right) \geq \varepsilon .
$$

On a alors :

$$
\xi \in B_{\theta, g f h}^{\varepsilon} \subset B_{\theta, g f h}^{\varpi} \text { et } f h \xi \in B_{\theta, g}^{\varpi}
$$

ou encore :

$$
g f h \xi \in(g f h) B_{\theta, g f h}^{\varpi} \cap g B_{\theta, g}^{\varpi}
$$

et, par conséquent, d'après la proposition 2.3.7, $g \in k_{g f h} z_{g} M$.

\subsubsection{Produit générique dans $\Gamma$}

Les lemmes 2.3.3 et 2.3.8 et la proposition 2.3.4 nous permettent maintenant de conclure :

Démonstration de la proposition 2.3.1. D'après le théorème 2.2.6, on peut trouver un réel $C>0$ tel que, pour tout $\gamma$ dans $\Gamma$, pour tout $\alpha$ dans $\theta_{\Gamma}^{c}, \alpha(\mu(\gamma)) \leq C$.

La famille $\left(\rho_{\alpha}, V_{\alpha}\right)_{\alpha \in \theta_{\Gamma}}$ vérifie les hypothèses de la proposition 2.3.4. Il existe donc une partie finie $F$ de $\Gamma$ et un réel $r>0$ tels que, pour toute famille $\left(U_{\alpha}, W_{\alpha}\right)_{\alpha \in \theta_{\Gamma}}$ où, pour tout $\alpha$ dans $\theta_{\Gamma}, U_{\alpha}$ est une droite et $W_{\alpha}$ un hyperplan de $V_{\alpha}$, il existe $f$ dans $F$ tel que, pour tout $\alpha$ dans $\theta_{\Gamma}$,

$$
d\left(f U_{\alpha}, \mathbb{P}\left(W_{\alpha}\right)\right) \geq r .
$$

Soient $\gamma_{1}, \gamma_{2}$ dans $\Gamma$. On peut trouver un élément $f_{\gamma_{1}, \gamma_{2}}$ de $F$ tel que, pour tout $\alpha$ dans $\theta_{\Gamma}$, on ait :

$$
d\left(f_{\gamma_{1}, \gamma_{2}} V_{\alpha, \gamma_{2}}^{M}, \mathbb{P}\left(V_{\alpha, \gamma_{1}}^{m}\right)\right) \geq r .
$$

En d'autres termes, le triplet $(g, h, f)=\left(\gamma_{1}, \gamma_{2}, f_{\gamma_{1}, \gamma_{2}}\right)$ vérifie les hypothèses des lemmes 2.3.3 et 2.3.8.

On pose $\pi\left(\gamma_{1}, \gamma_{2}\right)=\gamma_{1} f_{\gamma_{1}, \gamma_{2}} \gamma_{2}$. Comme $\mu(\Gamma)$ est à distance bornée de $E_{\theta_{\Gamma}}$, il existe, d'après le lemme 2.3.3, un réel $\kappa \geq 0$ tel que, pour tous $\gamma_{1}, \gamma_{2}$ dans $\Gamma$,

$$
\left\|\mu\left(\pi\left(\gamma_{1}, \gamma_{2}\right)\right)-\mu\left(\gamma_{1}\right)-\mu\left(\gamma_{2}\right)\right\| \leq \kappa .
$$


Par ailleurs, d'après le lemme 2.3.8, il existe une partie compacte $M$ de $G$ telle que, pour tous $\gamma_{1}, \gamma_{2}$ dans $\Gamma$, on ait $\gamma_{1} \in k_{\pi\left(\gamma_{1}, \gamma_{2}\right)} z_{\gamma_{1}} M$. En particulier, soient $R \geq 0$ et $\gamma_{1}, \gamma_{2}, \gamma_{1}^{\prime}, \gamma_{2}^{\prime}$ dans $\Gamma$, avec $\left\|\mu\left(\gamma_{1}\right)-\mu\left(\gamma_{1}^{\prime}\right)\right\| \leq R,\left\|\mu\left(\gamma_{2}\right)-\mu\left(\gamma_{2}^{\prime}\right)\right\| \leq R$ et $\pi\left(\gamma_{1}, \gamma_{2}\right)=\pi\left(\gamma_{1}^{\prime}, \gamma_{2}^{\prime}\right)=\gamma_{3}$. On a :

$$
\gamma_{1} \in k_{\gamma_{3}} z_{\gamma_{1}} M \text { et } \gamma_{1}^{\prime} \in k_{\gamma_{3}} z_{\gamma_{1}^{\prime}} M \text {. }
$$

Il vient

$$
\gamma_{1}^{-1} \gamma_{1}^{\prime} \in M^{-1} \nu^{-1}(b(0, R)) M
$$

L'ensemble $H=M^{-1} \nu^{-1}(b(0, R)) M \cap \Gamma$ est fini et l'on a :

$$
\gamma_{2}^{\prime}=f_{\gamma_{1}^{\prime}, \gamma_{2}^{\prime}}^{-1}\left(\gamma_{1}^{\prime}\right)^{-1} \gamma_{3} \in F^{-1} H^{-1} F \gamma_{2}
$$

\section{Mesures coniques}

Cette partie est indépendant de la première. Nous y traitons d'un point de vue abstrait le problème du calcul de l'exposant de convergence d'une mesure de Radon sur un espace vectoriel. Nous restreignons ensuite notre attention à certaines classes de mesures : si $\Gamma$ est un sous-groupe discret Zariski dense de $G$, la mesure de comptage $\sum_{\gamma \in \Gamma} \delta_{\mu(\gamma)}$ vérifiera les hypothèses que nous ferons.

Soit $\mathcal{E}$ un $\mathbb{R}$-espace vectoriel de dimension finie $r$ et $\nu$ une mesure de Radon positive sur $\mathcal{E}$.

\subsection{Divergence exponentielle}

Dans cette section, nous abordons d'un point de vue général l'étude des exposants de convergence de $\nu$. Nous commençons par donner des méthodes de calcul du type de la formule de Hadamard. Nous associons alors à $\nu$ une fonction homogène $\psi_{\nu}$ qui contient toutes les informations sur la divergence exponentielle de $\nu$ dans chacune des directions de $\mathcal{E}$. Enfin, nous introduisons un vocabulaire pour l'étude particulière des exposants de convergence associés aux formes linéaires de $\mathcal{E}$.

\subsubsection{Exposants de convergence et formules de Hadamard}

Étant donnée une norme $N$ sur $\mathcal{E}$, pour tout réel $t$, on pose :

$$
\mathcal{L}_{\nu}^{N}(t)=\int_{\mathcal{E}} e^{-t N(x)} \mathrm{d} \nu(x)
$$

et :

$$
\begin{aligned}
\tau_{\nu}^{N} & =\inf \left\{t \in \mathbb{R} \mid \mathcal{L}_{\nu}^{N}(t)<\infty\right\} \\
& =\sup \left\{t \in \mathbb{R} \mid \mathcal{L}_{\nu}^{N}(t)=\infty\right\} \in \mathbb{R} \cup\{+\infty,-\infty\}
\end{aligned}
$$


On l'appelle exposant de convergence de $\nu$ relativement à $N$.

Pour tous $x$ dans $\mathcal{E}$ et $a \leq b$ dans $\mathbb{R}$, on note $b^{N}(x, a)$ la boule de centre $x$ et de rayon $a$ relativement à la norme $N$ et $C^{N}(a, b)$ la couronne :

$$
\{x \in \mathcal{E} \mid a \leq N(x) \leq b\} .
$$

Donnons quelques formules de Hadamard pour le calcul de $\tau_{\nu}^{N}$ :

Lemme 3.1.1. Pour tous $a>0$ et $b, c \geq 0$ avec $b+c \geq a$, on $a$ :

$$
\tau_{\nu}^{N}=\frac{1}{a} \limsup _{n \rightarrow \infty} \frac{\log \left(\nu\left(C^{N}(n a-b, n a+c)\right)\right)}{n} .
$$

Pour tous $0<a<b$ et $c, d \geq 0$, on $a$ :

$$
\min \left(a \tau_{\nu}^{N}, b \tau_{\nu}^{N}\right) \leq \limsup _{n \rightarrow \infty} \frac{\log \left(\nu\left(C^{N}(n a-c, n b+d)\right)\right)}{n} \leq \max \left(a \tau_{\nu}^{N}, b \tau_{\nu}^{N}\right) .
$$

Si $\tau_{\nu}^{N}>0$, on $a$ :

$$
\tau_{\nu}^{N}=\limsup _{a \rightarrow \infty} \frac{\log \left(\nu\left(b^{N}(0, a)\right)\right)}{a} .
$$

Démonstration. Soient $a>0$ et $b, c \geq 0$ avec $b+c \geq a$. Il existe un entier $n_{0}>0$ tel que, pour tout $x$ dans $\mathcal{E}$,

$$
0<\operatorname{card}\left\{n \in \mathbb{N} \mid x \in C^{N}(n a-b, n a+c)\right\} \leq n_{0} .
$$

Il vient, pour tout $t$ dans $\mathbb{R}$,

$$
\begin{aligned}
\frac{1}{n_{0}} e^{-|t| \max (b, c)} \sum_{n=0}^{\infty} \nu\left(\left(C^{N}(n a\right.\right. & -b, n a+c)) e^{-t n a} \leq \int_{\mathcal{E}} e^{-t N(x)} \mathrm{d} \nu(x) \\
& \leq e^{|t| \max (b, c)} \sum_{n=0}^{\infty} \nu\left(\left(C^{N}(n a-b, n a+c)\right) e^{-t n a},\right.
\end{aligned}
$$

d'où la première formule.

Soient $0<a<b$ et $c, d \geq 0$. Comme $a<b$, il existe $R \geq 0$ tel que

$$
\mathcal{E}-b^{N}(0, R) \subset \bigcup_{n>0} C^{N}(n a-c, n b+d) .
$$

D'autre part, pour tout entier $n>0$, pour tout $x$ dans $C^{N}(n a-c, n b+d)$, pour tout $m>0$, on a :

$$
\left(x \in C^{N}(m a-c, m b+d)\right) \Rightarrow(m a-c \leq n b+d) \Rightarrow\left(m \leq \frac{n b+c+d}{a}\right)
$$

et, donc,

$$
\operatorname{card}\left\{m \in \mathbb{N}^{*} \mid x \in C^{N}(m a-c, m b+d)\right\} \leq \frac{n b+c+d}{a}
$$


Il vient, par conséquent, pour tout $t$ dans $\mathbb{R}$,

$$
\begin{aligned}
& a e^{-|t| \max (c, d)} \sum_{n=1}^{\infty} \frac{1}{n b+c+d} \nu\left(C^{N}(n a-c, n b+d)\right) e^{-n \max (a t, b t)} \\
& \leq \int_{\mathcal{E}-b^{N}(0, R)} e^{-t N(x)} \mathrm{d} \nu(x) \\
& \leq e^{|t| \max (c, d)} \sum_{n=1}^{\infty} \nu\left(C^{N}(n a-c, n b+d)\right) e^{-n \min (a t, b t)},
\end{aligned}
$$

d'où la deuxième formule.

Enfin, on a :

$$
\begin{aligned}
\tau_{\nu}^{N}=\limsup _{n \rightarrow \infty} \frac{\log \left(\nu\left(C^{N}(n-1, n)\right)\right)}{n} & \leq \limsup _{n \rightarrow \infty} \frac{\log \left(\nu\left(b^{N}(0, n)\right)\right)}{n} \\
& \leq \limsup _{a \rightarrow \infty} \frac{\log \left(\nu\left(b^{N}(0, a)\right)\right)}{a} .
\end{aligned}
$$

Supposons $\tau_{\nu}^{N}>0$. Soient $t$ et $s$ avec

$$
0<t<s<\limsup _{a \rightarrow \infty} \frac{\log \left(\nu\left(b^{N}(0, a)\right)\right)}{a} .
$$

Pour tout réel $a \geq 0$, il existe $b \geq a$ tel que, pour tout $c \geq b$, on ait :

$$
e^{s c}-e^{t c} \geq \nu\left(b^{N}(0, a)\right)
$$

et, donc, il existe $c \geq b$ tel que l'on ait :

$$
\nu\left(C^{N}(a, c)\right) \geq \nu\left(b^{N}(0, c)\right)-\nu\left(b^{N}(0, a)\right) \geq e^{s c}-\nu\left(b^{N}(0, a)\right) \geq e^{t c} .
$$

On peut donc construire une suite $\left(a_{n}\right)_{n \in \mathbb{N}}$ de réels $\geq 0$ avec, pour tout $n$ dans $\mathbb{N}$,

$$
a_{n+1} \geq a_{n}+1 \text { et } \nu\left(C^{N}\left(a_{n}+1, a_{n+1}\right)\right) \geq e^{t a_{n+1}} .
$$

Il vient alors :

$$
\int_{\mathcal{E}} e^{-t N(x)} \mathrm{d} \nu(x) \geq \sum_{n=0}^{\infty} \nu\left(C^{N}\left(a_{n}+1, a_{n+1}\right)\right) e^{-t a_{n+1}}=\infty,
$$

donc $t \leq \tau_{\nu}^{N}$, d'où la troisième formule.

De ces formules, on déduit immédiatement :

Corollaire 3.1.2. Soient $\nu$ et $\nu^{\prime}$ des mesures de Radon sur $\mathcal{E}$. S'il existe une partie compacte $M$ de $\mathcal{E}$ et un réel $\omega \geq 0$ tels que, pour tout borélien $B$ de $\mathcal{E}$,

$$
\nu^{\prime}(B) \leq \omega \nu(B+M)
$$

alors, pour toute norme $N$ sur $\mathcal{E}$, on a $\tau_{\nu^{\prime}}^{N} \leq \tau_{\nu}^{N}$. 


\subsubsection{Indicateur de croissance}

Soit $N$ une norme $\operatorname{sur} \mathcal{E}$. Soit $\mathcal{C} \subset \mathcal{E}$ un cône ouvert. On note $\tau_{\mathcal{C}, \nu}^{N}$ l'exposant de convergence relativement à $N$ de la mesure $\nu_{\mid \mathcal{C}}$.

Pour tout $x$ dans $\mathcal{E}-\{0\}$, on pose

$$
\psi_{\nu}(x)=N(x) \inf \tau_{\mathcal{C}, \nu}^{N}
$$

la borne inférieure étant prise sur l'ensemble des cônes ouverts $\mathcal{C}$ de $\mathcal{E}$ contenant $x$ et on pose $\psi_{\nu}(0)=0$. Cette fonction ne dépend pas de $N$. On l'appelle indicateur de croissance de $\nu$. Elle est positivement homogène, i.e. pour tous $t \geq 0$ et $x$ dans $\mathcal{E}$, on a $\psi_{\nu}(t x)=t \psi_{\nu}(x)$.

La fonction $\psi_{\nu}$ permet de calculer tous les exposants de convergence de $\nu$ :

Lemme 3.1.3. Soit $\theta: \mathcal{E} \rightarrow \mathbb{R}$ une fonction homogène et continue.

Si, pour tout $x$ dans $\mathcal{E}-\{0\}, \theta(x)>\psi_{\nu}(x)$, alors on a:

$$
\int_{\mathcal{E}} e^{-\theta(x)} \mathrm{d} \nu(x)<\infty
$$

$S^{\prime}$ 'il existe un $x$ dans $\mathcal{E}-\{0\}$ tel que $\theta(x)<\psi_{\nu}(x)$, alors on a :

$$
\int_{\mathcal{E}} e^{-\theta(x)} \mathrm{d} \nu(x)=\infty
$$

Démonstration. Soit $N$ une norme sur $\mathcal{E}$.

Supposons que, pour tout $x$ dans $\mathcal{E}-\{0\}, \theta(x)>\psi_{\nu}(x)$. En particulier, pour tout $x$ dans $\mathcal{E}, \psi_{\nu}(x)<\infty$. Soit $x$ dans $\mathcal{E}-\{0\}$. Il existe un cône ouvert $\mathcal{C}_{x}$ contenant $x$ et un réel $t_{x}$ tels que

$$
N(x) \tau_{\mathcal{C}_{x}, \nu}^{N}<N(x) t_{x}<\theta(x) .
$$

Il existe un cône ouvert $\mathcal{D}_{x} \subset \mathcal{C}_{x}$ contenant $x$ et tel que, pour tout $y \neq 0$ dans $\mathcal{D}_{x}$, on ait :

$$
N(y) t_{x}<\theta(y)
$$

et, donc,

$$
N(y) \tau_{\mathcal{D}_{x}, \nu}^{N} \leq N(y) \tau_{\mathcal{C}_{x}, \nu}^{N}<N(y) t_{x}<\theta(y) .
$$

Soient $x_{1}, \ldots, x_{n}$ dans $\mathcal{E}$ tels que l'on ait :

$$
\mathcal{E}-\{0\} \subset \mathcal{D}_{x_{1}} \cup \ldots \cup \mathcal{D}_{x_{n}} .
$$

Il vient :

$$
\int_{\mathcal{E}-\{0\}} e^{-\theta(x)} \mathrm{d} \nu(x) \leq \sum_{i=1}^{n} \int_{\mathcal{D}_{x_{i}}} e^{-t_{x_{i}} N(y)} \mathrm{d} \nu(y)<\infty .
$$

Supposons à présent qu'il existe $x$ dans $\mathcal{E}-\{0\}$ tel que $\theta(x)<\psi_{\nu}(x)$. On peut trouver un cône ouvert $\mathcal{C}$ de $\mathcal{E}$ et un réel $t$ tels que, pour tout $y \neq 0$ dans $\mathcal{C}$,

$$
\theta(y)<N(y) t<N(y) \frac{\psi_{\nu}(x)}{N(x)} \leq N(y) \tau_{\mathcal{C}, \nu}^{N} .
$$


Il vient :

$$
\int_{\mathcal{E}} e^{-\theta(y)} \mathrm{d} \nu(y) \geq \int_{\mathcal{C}} e^{-t N(y)} \mathrm{d} \nu(y)=\infty .
$$

Corollaire 3.1.4. Pour toute norme $N$ sur $\mathcal{E}$, on $a$ :

$$
\tau_{\nu}^{N}=\sup _{x \in \mathcal{E}-\{0\}} \frac{\psi_{\nu}(x)}{N(x)} .
$$

Corollaire 3.1.5. Pour toute fonction $\theta: \mathcal{E} \rightarrow \mathbb{R}$ homogène et continue, on $a$ :

$$
\psi_{e^{\theta} \nu}=\psi_{\nu}+\theta
$$

D'après le corollaire 3.1 .2 , on a :

Lemme 3.1.6. Soient $\nu$ et $\nu^{\prime}$ des mesures de Radon sur $\mathcal{E}$. S'il existe une partie compacte $M$ de $\mathcal{E}$ et un réel $\omega \geq 0$ tels que, pour tout borélien $B$ de $\mathcal{E}$,

$$
\nu^{\prime}(B) \leq \omega \nu(B+M)
$$

alors $\psi_{\nu^{\prime}} \leq \psi_{\nu}$

On suppose dorénavant qu'il existe une norme $N$ sur $\mathcal{E}$ pour laquelle $\tau_{\nu}^{N}<\infty$, c'est-à-dire que, pour toute norme $N$ sur $\mathcal{E}, \tau_{\nu}^{N}<\infty$.

Lemme 3.1.7. La fonction $\psi_{\nu}: \mathcal{E} \rightarrow \mathbb{R} \cup\{-\infty\}$ est semi-continue supérieurement.

Démonstration. Soit $N$ une norme sur $\mathcal{E}$.

Soit $x \neq 0$ dans $\mathcal{E}$ et $\left(x_{n}\right)_{n \in \mathbb{N}}$ une suite tendant vers $x$. Soit $\mathcal{C}$ un cône ouvert contenant $x$. Pour $n$ suffisament grand, $x_{n}$ appartient aussi à $\mathcal{C}$, et, donc, on a :

$$
\psi_{\nu}\left(x_{n}\right) \leq N\left(x_{n}\right) \tau_{\mathcal{C}, \nu}^{N}
$$

Il vient :

$$
\limsup _{n \rightarrow \infty} \psi_{\nu}\left(x_{n}\right) \leq \limsup _{n \rightarrow \infty}\left(N\left(x_{n}\right) \tau_{\mathcal{C}, \nu}^{N}\right)=N(x) \tau_{\mathcal{C}, \nu}^{N} .
$$

Par conséquent, on a :

$$
\limsup _{n \rightarrow \infty} \psi_{\nu}\left(x_{n}\right) \leq \psi_{\nu}(x)
$$

et, donc, $\psi_{\nu}$ est semi-continue supérieurement en $x$.

En particulier, il existe un réel $M$ tel que, pour tout $x$ dans $\mathcal{E}$ avec $N(x)=1$, $\psi_{\nu}(x) \leq M$. On a alors :

$$
\limsup _{y \in \mathcal{E}, y \rightarrow 0} \psi_{\nu}(y) \leq \limsup _{t \in \mathbb{R}_{+}, t \rightarrow 0}(t M)=0=\psi_{\nu}(0)
$$

et, donc, $\psi_{\nu}$ est semi-continue supérieurement en 0. 


\subsubsection{Convergence suivant les hyperplans}

Pour toute forme linéaire $\varphi$ dans $\mathcal{E}^{*}$, on pose :

$$
\mathcal{L}_{\nu}(\varphi)=\int_{\mathcal{E}} e^{-\varphi(x)} \mathrm{d} \nu(x)
$$

D'après l'inégalité de Hölder, l'ensemble $\left\{\varphi \in \mathcal{E}^{*}, \mathcal{L}_{\nu}(\varphi)<\infty\right\}$ est convexe.

Si $N$ est une norme sur $\mathcal{E}$, on pose

$$
\sigma_{\nu}^{N}=\inf _{\substack{\varphi \in \mathcal{E}^{*} \\ \mathcal{L}_{\nu}(\varphi)<\infty}} N(\varphi) .
$$

Pour toute forme linéaire $\varphi$ dans $\mathcal{E}^{*}$, on a :

$$
\int_{\mathcal{E}} e^{-\varphi(x)} \mathrm{d} \nu(x) \geq \int_{\mathcal{E}} e^{-N(\varphi) N(x)} \mathrm{d} \nu(x)
$$

et, donc, $\sigma_{\nu}^{N} \geq \tau_{\nu}^{N}$.

Proposition 3.1.8. S'il existe une forme linéaire $\varphi$ dans $\mathcal{E}^{*}$ telle que, pour tout $x$ dans $\mathcal{E}-\{0\}, \varphi(x)>\psi_{\nu}(x)$, alors, pour toute norme $N$ sur $\mathcal{E}$, on a :

$$
\sigma_{\nu}^{N}=\inf _{\substack{\varphi \in \mathcal{E}^{*} \\ \varphi \geq \psi_{\nu}}} N(\varphi) .
$$

Démonstration. D'après le lemme 3.1.3, on a toujours :

$$
\sigma_{\nu}^{N} \geq \inf _{\substack{\varphi \in \mathcal{E}^{*} \\ \varphi \geq \psi_{\nu}}} N(\varphi)
$$

Réciproquement, soit $\varphi_{0}$ une forme linéaire majorant strictement $\psi_{\nu}$ en dehors de 0 . Alors, pour toute forme linéaire $\varphi \geq \psi_{\nu}$, pour tout $t$ dans $\left.] 0,1\right], t \varphi_{0}+(1-t) \varphi$ majore strictement $\psi_{\nu}$ en dehors de 0 . Par conséquent, d'après le lemme 3.1.3, on a :

$$
\mathcal{L}_{\nu}\left(t \varphi_{0}+(1-t) \varphi\right)<\infty
$$

et, donc,

$$
N\left(t \varphi_{0}+(1-t) \varphi\right) \geq \sigma_{\nu}^{N}
$$

Il vient, quand $t \rightarrow 0$,

$$
N(\varphi) \geq \sigma_{\nu}^{N}
$$

ce qu'il fallait démontrer.

\subsection{Mesures à croissance concave}

Soit toujours $N$ une norme sur $\mathcal{E}$. Nous dirons que $\nu$ est à croissance concave si et seulement s'il existe des réels $\alpha, \beta, \gamma>0$ tels que, pour tous $x, y$ dans $\mathcal{E}$,

$$
\nu\left(b^{N}(x+y, \alpha)\right) \geq \gamma \nu\left(b^{N}(x, \beta)\right) \nu\left(b^{N}(y, \beta)\right) .
$$


Cette condition ne dépend pas de la norme choisie.

Dans cette section, nous allons démontrer :

Théorème 3.2.1. Si $\nu$ est à croissance concave, son indicateur de croissance est concave.

Le lecteur non intéressé par la démonstration de ce théorème peut directement passer à la section 3.3.

\subsection{1. Évaluations préliminaires}

Commençons par donner un lemme évident de recouvrement :

Lemme 3.2.2. Soient $N$ une norme sur $\mathcal{E}$ et $\beta>0$. Il existe un réel $M>0$ telle que, pour tout $x$ dans $\mathcal{E}$ et pour tout $a>0$, il existe un entier

$$
p \leq M(1+a)^{r}
$$

et des points $x_{1}, \ldots, x_{p}$ de $\mathcal{E}$ avec

$$
b^{N}(x, a) \subset \bigcup_{i=1}^{p} b^{N}\left(x_{i}, \beta\right)
$$

L'idée générale de nos preuves est d'utiliser le lemme 3.2.2 pour estimer la mesure d'une couronne $C^{N}(a, b), 0 \leq a \leq b$, à un facteur $(b+1)^{r}$ près, à l'aide de la mesure d'une boule $b^{N}(x, \beta)$, pour un certain $x$. Le facteur $(b+1)^{r}$ ne jouera pas de rôle du point de vue de la divergence exponentielle.

Nous commençons par itérer la formule de définition :

Lemme 3.2.3. Supposons $\nu$ à croissance concave. Soit $N$ une norme sur $\mathcal{E}$ et soient $\alpha, \beta, \gamma$ tels que, pour tous $x, y$ dans $E$,

$$
\nu\left(b^{N}(x+y, \alpha)\right) \geq \gamma \nu\left(b^{N}(x, \beta)\right) \nu\left(b^{N}(y, \beta)\right) .
$$

Alors il existe des réels $\theta>0$ et $\eta>0$ tels que, pour tout entier $k \geq 2$ et pour tous $x_{1}, \ldots, x_{k}$ dans $\mathcal{E}$, on ait :

$$
\begin{array}{r}
\nu\left(b^{N}\left(x_{1}+\ldots+x_{k},(k-1) \alpha+(k-2) \beta\right)\right) \\
\geq \theta \eta^{k} \nu\left(b^{N}\left(x_{1}, \beta\right)\right) \ldots \nu\left(b^{N}\left(x_{k}, \beta\right)\right) .
\end{array}
$$

Démonstration. Soit $N$ une norme sur $\mathcal{E}$. Donnons-nous $\alpha, \beta, \gamma$ comme ci-dessus et $M$ comme dans le lemme 3.2.2.

Posons

$$
\eta=\frac{1}{M(\alpha+\beta+1)^{r}}
$$

et montrons par récurrence sur $k \geq 2$ que, si $l$ est le plus petit entier tel que $2^{l} \geq k$, 
alors, pour tous $x_{1}, \ldots, x_{k}$ dans $\mathcal{E}$, on a :

$$
\begin{aligned}
& \nu\left(b^{N}\left(x_{1}+\ldots+x_{k},(k-1) \alpha+(k-2) \beta\right)\right) \\
& \geq \frac{\gamma^{k-1} \eta^{k-2}}{\left(2^{\left.2(l-1)+4(l-2)+8(l-3)+\ldots+2^{l-1}\right)^{r}} \nu\left(b^{N}\left(x_{1}, \beta\right)\right) \ldots \nu\left(b^{N}\left(x_{k}, \beta\right)\right),\right.},
\end{aligned}
$$

ce qui implique le lemme.

Pour $k=2$, il s'agit juste de la définition de la croissance concave.

Pour $k=3$, c'est un raisonnement analogue à celui fait ci-après.

Soit donc $k \geq 4$ et supposons la formule vraie pour tous les entiers $<k$. Soient $l$ le plus petit entier tel que $2^{l} \geq k$ et $x_{1}, \ldots, x_{k}$ dans $\mathcal{E}$.

Si $k$ est pair, on pose $h=\frac{k}{2}$; s'il est impair, on pose $h=\frac{k-1}{2}$. Dans les deux cas, on a

$$
2^{l-1} \geq h>2^{l-2} \text { et } 2^{l-1} \geq k-h>2^{l-2} .
$$

D'après le lemme 3.2.2, il existe un point $y_{1}$ dans $b^{N}\left(x_{1}+\ldots+x_{h},(h-1)(\alpha+\beta)\right)$ tel que l'on ait :

$$
\begin{aligned}
\nu\left(b ^ { N } \left(x_{1}+\ldots+x_{h},(h-1) \alpha+\right.\right. & (h-2) \beta)) \\
& \leq M(1+(h-1) \alpha+(h-2) \beta)^{r} \nu\left(b^{N}\left(y_{1}, \beta\right)\right) \\
& \leq \frac{2^{r(l-1)}}{\eta} .
\end{aligned}
$$

De même, il existe un point $y_{2}$ dans $b^{N}\left(x_{h+1}+\ldots+x_{k},(k-h-1)(\alpha+\beta)\right)$ tel que l'on ait :

$$
\begin{aligned}
\nu\left(b ^ { N } \left(x_{h+1}+\ldots+x_{k},\right.\right. & (k-h-1) \alpha+(k-h-2) \beta)) \\
& \leq M(1+(k-h-1) \alpha+(k-h-2) \beta)^{r} \nu\left(b^{N}\left(y_{2}, \beta\right)\right) \\
& \leq \frac{2^{r(l-1)}}{\eta} .
\end{aligned}
$$

On a alors :

$$
\begin{aligned}
\nu\left(b^{N}\left(y_{1}+y_{2}, \alpha\right)\right) \geq & \gamma \nu\left(b^{N}\left(y_{1}, \beta\right)\right) \nu\left(b^{N}\left(y_{2}, \beta\right)\right) \\
\geq & \frac{\gamma \eta^{2}}{2^{2 r(l-1)}} \nu\left(b^{N}\left(x_{1}+\ldots+x_{h},(h-1) \alpha+(k-2) \beta\right)\right) \\
& \nu\left(b^{N}\left(x_{h+1}+\ldots+x_{k},(k-h-1) \alpha+(k-h-2) \beta\right)\right) .
\end{aligned}
$$

Donc, par récurrence,

$$
\begin{aligned}
& \nu\left(b^{N}\left(y_{1}+y_{2}, \alpha\right)\right) \\
& \quad \geq \frac{\gamma^{k-1} \eta^{k-2}}{\left(2^{\left.2(l-1)+4(l-2)+8(l-3)+\ldots+2^{l-1}\right)^{r}} \nu\left(b^{N}\left(x_{1}, \beta\right)\right) \ldots \nu\left(b^{N}\left(x_{k}, \beta\right)\right),\right.},
\end{aligned}
$$

d'où le résultat, puisque l'on a :

$$
b^{N}\left(y_{1}+y_{2}, \alpha\right) \subset b^{N}\left(x_{1}+\ldots+x_{k},(k-1) \alpha+(k-2) \beta\right) .
$$


Par récurrence, la formule est vraie pour tout $k \geq 2$.

Rappelons que, si $\left(t_{n}\right)_{n \in \mathbb{N}}$ est une suite de nombres réels avec, pour tous $n, p$ dans $\mathbb{N}, t_{n+p} \geq t_{n}+t_{p}$, alors la suite $\left(\frac{1}{n} t_{n}\right)_{n \geq 1}$ converge dans $\mathbb{R} \cup\{-\infty\}$. Les deux lemmes qui suivent s'inspirent de la démonstration de ce résultat usuel pour minorer la limite inférieure du logarithme de la mesure des couronnes.

Lemme 3.2.4. Supposons $\nu$ à croissance concave. Soit $N$ une norme sur $\mathcal{E}$. Il existe des réels $\theta, \eta, \kappa>0$ ayant la propriété suivante : pour tout cône ouvert $\mathcal{C}$ de $\mathcal{E}$, pour tout $x \neq 0$ dans $\mathcal{C}$ et pour tout $\varepsilon>0$, il existe un cône ouvert $\mathcal{D} \subset \mathcal{C}$ contenant $x$ et un réel $a_{0}>0$ tels que, pour tous réels $a \geq a_{0}$ et $b \geq 0$, pour tout entier naturel $m$ et pour toute partition $m=n_{1}+\ldots+n_{p}$ de $m$, on ait :

$$
\begin{aligned}
\nu\left(C^{N}((1-\varepsilon) m a-\right. & (p-1) \kappa, m a+p b+(p-1) \kappa) \cap \mathcal{C}) \\
& \geq \frac{\theta \eta^{p}}{\left(\prod_{k=1}^{p}\left(1+n_{k} a+b\right)\right)^{r}} \prod_{k=1}^{p} \nu\left(C^{N}\left(n_{k} a, n_{k} a+b\right) \cap \mathcal{D}\right) .
\end{aligned}
$$

Démonstration. Il suffit de montrer la propriété pour des partitions $m=n_{1}+\ldots+$ $n_{p}$ de l'entier $m$ avec $p \geq 2$.

Soit $N$ une norme sur $\mathcal{E}, \alpha, \beta, \gamma$ comme dans la définition de la croissance concave, $M$ comme dans le lemme 3.2.2 et $\theta, \eta$ comme dans le lemme 3.2.3.

Soit $\mathcal{C}$ un cône ouvert de $\mathcal{E}, x$ un vecteur unitaire de $\mathcal{C}$ et $0<\varepsilon<1$. Il existe un cône ouvert convexe $\mathcal{D}$ de $\mathcal{E}$ contenant $x$ tel que $\overline{\mathcal{D}}-\{0\} \subset \mathcal{C}$ et que, pour tout vecteur unitaire $y$ de $\mathcal{D}, N(y-x) \leq \varepsilon$.

Soient $x_{1}, \ldots, x_{p}$, des vecteurs de $\mathcal{D}$. Pour tout $i$ dans $\llbracket 1, p \rrbracket$, on a :

$$
N\left(x_{i}-N\left(x_{i}\right) x\right) \leq \varepsilon N\left(x_{i}\right)
$$

et, donc :

$$
N\left(\left(x_{1}+\ldots+x_{p}\right)-\left(N\left(x_{1}\right)+\ldots+N\left(x_{p}\right)\right) x\right) \leq \varepsilon\left(N\left(x_{1}\right)+\ldots+N\left(x_{p}\right)\right) .
$$

Il vient :

$$
N\left(x_{1}+\ldots+x_{p}\right) \geq(1-\varepsilon)\left(N\left(x_{1}\right)+\ldots+N\left(x_{p}\right)\right) .
$$

Il existe un réel $a_{0}>0$ tel que, pour tout $y$ dans $\mathcal{D}$ avec $N(y) \geq 1-\varepsilon$, on ait :

$$
b^{N}\left(y, \frac{\alpha+2 \beta}{a_{0}}\right) \subset \mathcal{C} .
$$

Alors, pour tout $a \geq a_{0}$, pour tout $b \geq 0$ et pour tout entier $n$, on ait :

$$
b^{N}\left(C^{N}((1-\varepsilon) n a, n a+b) \cap \mathcal{D},(\alpha+2 \beta) n\right) \subset \mathcal{C} .
$$

Soient $a \geq a_{0}$ et $b \geq 0$. Pour tout entier $n$, choisissons un point $x_{n}$ dans $b^{N}\left(C^{N}(n a, n a+b) \cap \mathcal{D}, \bar{\beta}\right)$ tel que l'on ait :

$$
\nu\left(b^{N}\left(x_{n}, \beta\right)\right) \geq \frac{1}{M(1+n a+b)^{r}} \nu\left(C^{N}(n a, n a+b) \cap \mathcal{D}\right) .
$$


Soit $m$ un entier naturel et $m=n_{1}+\ldots+n_{p}$ une partition de $m$ avec $p \geq 2$. On a :

$$
\begin{aligned}
\nu\left(b ^ { N } \left(x_{n_{1}}+\ldots+\right.\right. & \left.\left.x_{n_{p}},(p-1) \alpha+(p-2) \beta\right)\right) \\
& \geq \theta \eta^{p} \prod_{k=1}^{p} \nu\left(b^{N}\left(x_{n_{k}}, \beta\right)\right) \\
& \geq \frac{\theta \eta^{p}}{M^{p}\left(\prod_{k=1}^{p}\left(1+n_{k} a+b\right)\right)^{r}} \prod_{k=1}^{p} \nu\left(C^{N}\left(n_{k} a, n_{k} a+b\right) \cap \mathcal{D}\right) .
\end{aligned}
$$

Or, on a :

$$
x_{n_{1}}+\ldots+x_{n_{p}} \in b^{N}\left(C^{N}((1-\varepsilon) m a, m a+p b) \cap \mathcal{D}, p \beta\right)
$$

et, donc, d'une part,

$$
\begin{aligned}
b^{N}\left(x_{n_{1}}+\ldots+x_{n_{p}},\right. & (p-1) \alpha+(p-2) \beta) \\
& \subset b^{N}\left(C^{N}((1-\varepsilon) m a, m a+p b) \cap \mathcal{D},(p-1)(\alpha+2 \beta)\right) \subset \mathcal{C}
\end{aligned}
$$

et, d'autre part,

$$
\begin{aligned}
b^{N}\left(x_{n_{1}}+\ldots+\right. & \left.x_{n_{p}},(p-1) \alpha+(p-2) \beta\right) \\
& \subset C^{N}((1-\varepsilon) m a-(p-1)(\alpha+\beta), m a+p b+(p-1)(\alpha+\beta)) .
\end{aligned}
$$

Il vient :

$$
\begin{aligned}
\nu\left(C^{N}((1-\varepsilon) m a\right. & -(p-1)(\alpha+\beta), m a+p b+(p-1)(\alpha+\beta)) \cap \mathcal{C}) \\
& \geq \frac{\theta \eta^{p}}{M^{p}\left(\prod_{k=1}^{p}\left(1+n_{k} a+b\right)\right)^{r}} \prod_{k=1}^{p} \nu\left(C^{N}\left(n_{k} a, n_{k} a+b\right) \cap \mathcal{D}\right) .
\end{aligned}
$$

Pour les mesures à croissance concave, on a donc un complément aux formules du lemme 3.1.1 :

Lemme 3.2.5. Supposons $\nu$ à croissance concave. Soit $N$ une norme sur $\mathcal{E}$. Pour tout cône ouvert $\mathcal{C}$ de $\mathcal{E}$, pour tout $x \neq 0$ dans $\mathcal{C}$ et pour tout $\varepsilon>0$, il existe $a_{0}>0$ tel que, pour tout $a \geq a_{0}$, on ait :

$$
\frac{1}{a} \liminf _{n \rightarrow \infty} \frac{\log \left(\nu\left(C^{N}(n(1-\varepsilon) a, n(1+\varepsilon) a) \cap \mathcal{C}\right)\right)}{n} \geq \frac{\psi_{\nu}(x)}{N(x)} .
$$

Démonstration. Soient $N$ une norme sur $\mathcal{E}$ et $\kappa, \theta, \eta$ comme dans le lemme 3.2.4. Soient $\mathcal{C}$ un cône ouvert de $\mathcal{E}, x$ un vecteur non nul de $\mathcal{C}$ et $\varepsilon>0$.

D'après le lemme 3.2.4, on peut trouver un cône ouvert $\mathcal{D} \subset \mathcal{C}$ contenant $x$ et un réel $a_{0}>0$ tel que, pour tout $a \geq a_{0}$, pour tous $m$ et $n$ entiers $\geq 1$, si 
$m=p n+q$ est la division euclidienne de $m$ par $n$, alors on a :

$$
\begin{gathered}
\nu\left(C^{N}((1-\varepsilon) m a-p \kappa,(m+p+1) a+p \kappa) \cap \mathcal{C}\right) \\
\geq \frac{\theta \eta^{p+1}}{\left((1+(n+1) a)^{p}(1+(q+1) a)\right)^{r}} \nu\left(C^{N}(n a,(n+1) a) \cap \mathcal{D}\right)^{p} \\
\nu\left(C^{N}(q a,(q+1) a) \cap \mathcal{D}\right) .
\end{gathered}
$$

Soit $n_{0}>0$ tel que

$$
\frac{\kappa}{a_{0} n_{0}} \leq \varepsilon \text { et que } \frac{1}{n_{0}} \leq \varepsilon
$$

Soit $a \geq a_{0}$. Pour tous $m, n \geq n_{0}$, si $m=p n+q$ est la division euclidienne de $m$ par $n$, on a :

$$
\begin{array}{r}
\nu\left(C^{N}(m(1-3 \varepsilon) a, m(1+3 \varepsilon) a) \cap \mathcal{C}\right) \geq \frac{\theta \eta^{p+1}}{\left((1+(n+1) a)^{p}(1+(q+1) a)\right)^{r}} \\
\nu\left(C^{N}(n a,(n+1) a) \cap \mathcal{D}\right)^{p} \nu\left(C^{N}(q a,(q+1) a) \cap \mathcal{D}\right)
\end{array}
$$

et, donc, pour tout $n \geq n_{0}$,

$$
\begin{aligned}
& \frac{1}{a} \liminf _{m \rightarrow \infty} \frac{\log \left(\nu\left(C^{N}(m(1-3 \varepsilon) a, m(1+3 \varepsilon) a) \cap \mathcal{C}\right)\right)}{m} \\
& \geq \frac{1}{n a} \log \left(\frac{\eta}{(1+(n+1) a)^{r}}\right)+\frac{\log \left(\nu\left(C^{N}(n a,(n+1) a) \cap \mathcal{D}\right)\right)}{n a} .
\end{aligned}
$$

Il vient, en faisant tendre $n$ vers $\infty$, d'après le lemme 3.1.1,

$$
\frac{1}{a} \liminf _{m \rightarrow \infty} \frac{\log \left(\nu\left(C^{N}(m(1-3 \varepsilon) a, m(1+3 \varepsilon) a) \cap \mathcal{C}\right)\right)}{m} \geq \tau_{\mathcal{D}, \nu}^{N} \geq \frac{\psi_{\nu}(x)}{N(x)} .
$$

\subsubsection{Démonstration du théorème de concavité}

Nous pouvons à présent conclure la preuve du théorème 3.2.1. Puisque $\psi_{\nu}$ est homogène, il s'agit de prouver qu'elle est sur-additive. L'idée consiste à se donner $x$ et $y$ dans $\mathcal{E}$, avec $x+y \neq 0$ et à estimer le volume d'une couronne dans un cône autour de $x+y$ à l'aide de $\psi_{\nu}(x)+\psi_{\nu}(y)$, en employant le lemme 3.2.5.

Démonstration du théorème 3.2.1. Soit $N$ une norme sur $\mathcal{E}$ et soient toujours $\alpha, \beta, \gamma>0$ tels que, pour tous $x, y$ dans $\mathcal{E}$,

$$
\nu\left(b^{N}(x+y, \alpha)\right) \geq \gamma \nu\left(b^{N}(x, \beta)\right) \nu\left(b^{N}(y, \beta)\right) .
$$

Soit $M$ comme dans le lemme 3.2.2.

Soient $x$ et $y$ des vecteurs non nuls de $\mathcal{E}$. Commençons par supposer $x+y \neq 0$. Soit $\mathcal{C}$ un cône ouvert contenant $x+y$.

Soient $0<\omega<\frac{1}{2}$ et $\mathcal{C}_{1}$ un cône ouvert de $\mathcal{E}$, contenant $x+y$ et tel que $\overline{\mathcal{C}_{1}}-\{0\} \subset \mathcal{C}$. On peut trouver un réel $\varepsilon>0$ et des cônes ouverts $\mathcal{A}$ et $\mathcal{B}$ contenant 
respectivement $x$ et $y$ tels que l'on ait

$$
\begin{aligned}
&\left(C^{N}(N(x)(1-\varepsilon),\right.N(x)(1+\varepsilon)) \cap \mathcal{A}) \\
&+\left(C^{N}(N(y)(1-\varepsilon), N(y)(1+\varepsilon)) \cap \mathcal{B}\right) \\
& \quad \subset\left(C^{N}(N(x+y)(1-\omega), N(x+y)(1+\omega)) \cap \mathcal{C}_{1}\right) .
\end{aligned}
$$

D'après le lemme 3.2.5, on peut trouver un réel $a>0$ tel que l'on ait :

$$
\frac{1}{a} \liminf _{n \rightarrow \infty} \frac{\log \left(\nu\left(C^{N}(n(1-\varepsilon) a N(x), n(1+\varepsilon) a N(x)) \cap \mathcal{A}\right)\right)}{n} \geq \psi_{\nu}(x)
$$

et de même en remplaçant $x$ par $y$.

D'après le lemme 3.2.2, pour tout entier $n$, il existe un vecteur $x_{n}$ de $\mathcal{E}$ tel que

$$
d\left(x_{n}, C^{N}(n a(1-\varepsilon) N(x), n a(1+\varepsilon) N(x)) \cap \mathcal{A}\right) \leq \beta
$$

et que

$$
\begin{aligned}
\nu\left(C^{N}(n a(1-\varepsilon) N(x), n a(1+\varepsilon) N(x))\right. & \cap \mathcal{A}) \\
\leq & M(1+n a(1+\varepsilon) N(x))^{r} \nu\left(b^{N}\left(x_{n}, \beta\right)\right)
\end{aligned}
$$

et un vecteur $y_{n}$ de $\mathcal{E}$ vérifiant les inégalités analogues en remplaçant $x$ par $y$.

Alors, pour tout entier $n>0$, le vecteur

$$
\frac{x_{n}+y_{n}}{n a}
$$

est à une distance $\leq \frac{2 \beta}{n a}$ de l'ensemble

$$
\left(C^{N}(N(x)(1-\varepsilon), N(x)(1+\varepsilon)) \cap \mathcal{A}\right)+\left(C^{N}(N(y)(1-\varepsilon), N(y)(1+\varepsilon)) \cap \mathcal{B}\right) .
$$

de sorte qu'il existe un entier $n_{0}>0$ tel que, pour tout $n \geq n_{0}$, on ait :

$$
b^{N}\left(x_{n}+y_{n}, \alpha\right) \subset C^{N}(n a(1-2 \omega) N(x+y), n a(1+2 \omega) N(x+y)) \cap \mathcal{C} .
$$

Il vient, pour tout entier $n \geq n_{0}$,

$$
\begin{aligned}
& \nu\left(C^{N}(n a(1-2 \omega) N(x+y),\right.n a(1+2 \omega) N(x+y)) \cap \mathcal{C}) \\
& \geq \nu\left(b^{N}\left(x_{n}+y_{n}, \alpha\right)\right) \geq \gamma \nu\left(b^{N}\left(x_{n}, \beta\right)\right) \nu\left(b^{N}\left(y_{n}, \beta\right)\right) \\
& \geq \frac{\gamma}{M^{2}(1+n a(1+\varepsilon) N(x))^{r}(1+n a(1+\varepsilon) N(y))^{r}} \\
& \nu\left(C^{N}(n a(1-\varepsilon) N(x), n a(1+\varepsilon) N(x)) \cap \mathcal{A}\right) \\
& \nu\left(C^{N}(n a(1-\varepsilon) N(y), n a(1+\varepsilon) N(y)) \cap \mathcal{B}\right) .
\end{aligned}
$$

En faisant tendre $n$ vers $\infty$ on a, d'après le lemme 3.1.1,

$$
\max \left(N(x+y)(1+2 \omega) \tau_{\mathcal{C}, \nu}^{N}, N(x+y)(1-2 \omega) \tau_{\mathcal{C}, \nu}^{N}\right) \geq \psi_{\nu}(x)+\psi_{\nu}(y) .
$$


Comme l'inégalité ci-dessus est vraie pour tout $0<\omega<\frac{1}{2}$, il vient :

$$
N(x+y) \tau_{\mathcal{C}, \nu}^{N} \geq \psi_{\nu}(x)+\psi_{\nu}(y),
$$

d'où :

$$
\psi_{\nu}(x+y) \geq \psi_{\nu}(x)+\psi_{\nu}(y)
$$

ce qu'il fallait démontrer.

Supposons à présent que $y=-x$. Pour tout $t>1$, on a :

$$
t x-x=(t-1) x \neq 0
$$

et, donc,

$$
\psi_{\nu}(t x)+\psi_{\nu}(-x) \leq \psi_{\nu}((t-1) x)
$$

et, donc, comme $\psi_{\nu}$ est homogène, on a bien, même quand $\psi_{\nu}(x)=-\infty$,

$$
\psi_{\nu}(x)+\psi_{\nu}(-x) \leq 0
$$

\subsection{Mesures à croissance concave divergente}

Nous dirons que $\nu$ est à croissance divergente (resp. strictement divergente) si et seulement si, étant donnée une norme $N$ sur $\mathcal{E}$, on a $\tau_{\nu}^{N} \geq 0\left(\right.$ resp. $\left.\tau_{\nu}^{N}>0\right)$. Ces conditions sont indépendantes de la norme choisie.

\subsubsection{Contrôle de la divergence}

Pour les mesures à croissance concave, on peut améliorer le lemme 3.1.1 :

Proposition 3.3.1. Si $\nu$ est à croissance concave strictement divergente, pour toute norme $N$ sur $\mathcal{E}$, on a :

$$
\frac{\log \left(\nu\left(b^{N}(0, a)\right)\right)}{a} \underset{a \rightarrow \infty}{\longrightarrow} \tau_{\nu}^{N} \text { et } \nu\left(b^{N}(0, a)\right) \underset{a \rightarrow \infty}{=} O\left(a^{r-1} e^{a \tau_{\nu}^{N}}\right) .
$$

La démonstration utilise le lemme suivant, dont la démonstration est analogue à celle du lemme 3.2.4, en employant une variante du lemme 3.2.2.

Lemme 3.3.2. Supposons $\nu$ à croissance concave. Soit $N$ une norme sur $\mathcal{E}$. Il existe des réels $\theta, \eta, \kappa>0$ tels que, pour tout réel $a \geq 0$ et pour tous réels $a_{1}, \ldots, a_{p}$ tels que $a=a_{1}+\ldots+a_{p}$, on ait :

$$
\begin{aligned}
\nu\left(b^{N}(0, a+p+(p-1) \kappa)\right) \\
\quad \geq \frac{\theta \eta^{p}}{\left(\left(1+a_{1}\right) \ldots\left(1+a_{p}\right)\right)^{r-1}} \nu\left(C^{N}\left(a_{1}, a_{1}+1\right)\right) \ldots \nu\left(C^{N}\left(a_{p}, a_{p}+1\right)\right) .
\end{aligned}
$$


Démonstration de la proposition 3.3.1. Commençons par remarquer que, si $f$ : $\mathbb{R}_{+} \rightarrow \mathbb{R}$ est une fonction croissante, on a :

$$
\limsup _{\substack{a \rightarrow \infty \\ a \in \mathbb{R}_{+}^{*}}} \frac{f(a)}{a}=\limsup _{\substack{n \rightarrow \infty \\ n \in \mathbb{N}^{*}}} \frac{f(n)}{n} \text { et } \liminf _{\substack{a \rightarrow \mathbb{R}_{+}^{*} \\ a \in \mathbb{R}_{+}^{*}}} \frac{f(a)}{a}=\liminf _{\substack{n \rightarrow \mathbb{N}^{*} \\ n \in \mathbb{N}^{*}}} \frac{f(n)}{n} .
$$

Soit alors $N$ une norme sur $\mathcal{E}$. D'après le lemme 3.1.1, on a :

$$
\tau_{\nu}^{N}=\limsup _{a \rightarrow \infty} \frac{\log \left(\nu\left(b^{N}(0, a)\right)\right)}{a} .
$$

Soient $\theta, \eta, \kappa>0$ comme dans le lemme 3.3.2. Soient $m \geq n \geq 1$ des entiers naturels et $m=p n+q$ la division euclidienne de $m$ par $n$. On a :

$$
\begin{aligned}
\nu\left(b^{N}\left(0, m\left(1+\frac{\kappa+2}{n}\right)\right)\right) \geq & \nu\left(b^{N}(0, m+(p+1)+p \kappa)\right) \\
\geq & \frac{\theta \eta^{p+1}}{\left((1+n)^{p}(1+q)\right)^{r-1}} \\
& \nu\left(C^{N}(n, n+1)\right)^{p} \nu\left(C^{N}(q, q+1)\right) .
\end{aligned}
$$

Il vient, pour tout $n \geq 1$, d'après la remarque ci-dessus,

$$
\begin{aligned}
\left(1+\frac{\kappa+2}{n}\right) \liminf _{a \rightarrow \infty} \frac{\log \left(\nu\left(b^{N}(0, a)\right)\right)}{a} \\
\geq\left(\frac{1}{n} \log \left(\frac{\eta}{(1+n)^{r-1}}\right)+\frac{\log \left(\nu\left(C^{N}(n, n+1)\right)\right)}{n}\right) .
\end{aligned}
$$

On en déduit, en réutilisant le lemme 3.1.1 :

$$
\liminf _{a \rightarrow \infty} \frac{\log \left(\nu\left(b^{N}(0, a)\right)\right)}{a} \geq \limsup _{n \rightarrow \infty} \frac{\log \left(\nu\left(C^{N}(n, n+1)\right)\right)}{n}=\tau_{\nu}^{N}
$$

et, donc,

$$
\frac{\log \left(\nu\left(b^{N}(0, a)\right)\right)}{a} \underset{a \rightarrow \infty}{\longrightarrow} \tau_{\nu}^{N} .
$$

Mais alors, pour tout $n \geq 1$, on a :

$$
(n+\kappa+2) \tau_{\nu}^{N} \geq \log \left(\frac{\eta}{(1+n)^{r-1}}\right)+\log \left(\nu\left(C^{N}(n, n+1)\right)\right)
$$

d'où

$$
\nu\left(C^{N}(n, n+1)\right) \leq \frac{1}{\eta}(1+n)^{r-1} e^{(n+\kappa+2) \tau_{\nu}^{N}} .
$$

En d'autres termes, il existe un réel $M \geq 0$ tel que, pour tout entier naturel $n$, on ait :

$$
\nu\left(C^{N}(n, n+1)\right) \leq M(1+n)^{r-1} e^{n \tau_{\nu}^{N}} .
$$


Il vient, comme $\tau_{\nu}^{N}>0$,

$$
\nu\left(b^{N}(0, n)\right) \leq M \sum_{k=0}^{n-1}(1+k)^{r-1} e^{k \tau_{\nu}^{N}} \underset{n \rightarrow \infty}{=} O\left(n^{r-1} e^{n \tau_{\nu}^{N}}\right) .
$$

On en déduit le résultat.

\subsubsection{Propriétés de concavité}

Les résultats suivants appliquent des propriétés élémentaires de concavité au calcul des exposants de convergence.

Proposition 3.3.3. Si $\nu$ est à croissance concave divergente et s'il existe une forme linéaire $\varphi$ dans $\mathcal{E}^{*}$ telle que, pour tout $x$ dans $\mathcal{E}-\{0\}, \varphi(x)>\psi_{\nu}(x)$, alors, pour toute norme $N$ sur $\mathcal{E}$, on a:

$$
\sigma_{\nu}^{N}=\tau_{\nu}^{N}
$$

Démonstration. C'est une conséquence du corollaire 3.1.4, de la proposition 3.1.8, du théorème 3.2.1 et du résultat de convexité ci-après.

Lemme 3.3.4. Soit $\psi: \mathcal{E} \rightarrow \mathbb{R} \cup\{-\infty\}$ une fonction concave homogène semicontinue supérieurement. Soient $N$ une norme sur $\mathcal{E}$ et

$$
\tau=\sup _{x \in \mathcal{E}-\{0\}} \frac{\psi(x)}{N(x)} .
$$

Si $\tau \geq 0$, il existe une forme linéaire $\varphi$ dans $\mathcal{E}^{*}$ telle que

$$
\varphi \geq \psi \text { et que } N(\varphi)=\tau \text {. }
$$

Démonstration. Si $\tau=0$, le résultat est clair. Supposons donc $\tau>0$ et, pour simplifier les notations, $\tau=1$.

Soit $x$ dans $\mathcal{E}$ tel que $N(x)=1$ et que $\psi(x)=1$. Pour tout $y$ dans $\mathcal{E}$, on a :

$$
\psi(y) \leq N(y)
$$

et, donc, les parties convexes fermées de $\mathbb{R} \times \mathcal{E}$

$$
\{(s, y) \in \mathbb{R} \times \mathcal{E} \mid s \geq N(y)\} \text { et }\{(t, z) \in \mathbb{R} \times \mathcal{E} \mid t \leq \psi(z)\}
$$

ne se rencontrent qu'en leurs bords. D'après le théorème de Hahn-Banach, il existe un réel $\alpha$ et une forme linéaire $\varphi$ dans $\mathcal{E}^{*}$ non tout deux nuls et tels que, pour tous $y, z$ dans $\mathcal{E}$ avec $\psi(z)>-\infty$, on ait :

$$
\alpha N(y)+\varphi(y) \geq \alpha \psi(z)+\varphi(z) .
$$

En particulier, pour tout $y$ dans $\mathcal{E}$, on a :

$$
\alpha N(y)+\varphi(y) \geq \alpha N(x)+\varphi(x) .
$$


Supposons $\alpha \leq 0$. Alors, pour tout $y$ dans $\mathcal{E}$, on a :

$$
\varphi(y) \geq \alpha+\varphi(x)
$$

et, donc, $\varphi=0$. Mais alors, $\alpha \neq 0$ et, pour tout $y$ dans $\mathcal{E}$, on a :

$$
N(y) \leq 1
$$

ce qui est absurde. Par conséquent, $\alpha>0$.

On peut donc supposer que $\alpha=1$. Pour tout $y$ dans $\mathcal{E}$, on a :

$$
\varphi(x-y) \leq N(y)-N(x) \leq N(y-x)
$$

donc $N(\varphi) \leq 1$. Mais, en posant $y=\frac{x}{2}$ ci-dessus, il vient :

$$
\varphi\left(\frac{x}{2}\right) \leq-\frac{1}{2}
$$

donc $\varphi(x)=-1$ et $N(\varphi)=1$.

Or, pour tout $z$ dans $\mathcal{E}$, on a :

$$
\psi(z)+\varphi(z) \leq N(x)+\varphi(x)=0
$$

i.e. $-\varphi \geq \psi$.

Corollaire 3.3.5. Supposons que $\nu$ est à croissance concave strictement divergente et qu'il existe une forme linéaire majorant strictement $\psi_{\nu}$ en dehors de 0. Soit $N$ la norme associée à un produit scalaire $\langle.,$.$\rangle sur \mathcal{E}$. Alors, il existe une unique forme linéaire $\varphi \geq \psi_{\nu}$ de norme $\tau_{\nu}^{N}$. Si $x$ est l'unique vecteur unitaire de $\mathcal{E}$ tel que $\psi_{\nu}(x)=\tau_{\nu}^{N}, \varphi$ est la forme $\left\langle\tau_{\nu}^{N} x,.\right\rangle$.

Démonstration. L'existence a été prouvée au corollaire 3.3.3.

Démontrons l'unicité. Soit $\varphi \geq \psi_{\nu}$ de norme $\tau_{\nu}^{N}$. Comme la norme euclidienne est strictement convexe, d'après le corollaire 3.1.4 et le théorème 3.2.1, il existe un unique vecteur unitaire $x$ de $\mathcal{E}$ tel que $\psi_{\nu}(x)=\tau_{\nu}^{N}$. On a :

$$
\varphi(x) \geq \psi_{\nu}(x)=\tau_{\nu}^{N}=N(\varphi) N(x)
$$

d'où le résultat, d'après le théorème de Cauchy-Schwarz.

\section{L'indicateur de croissance de $\Gamma$}

Dans cette partie, nous utilisons l'existence d'un produit générique dans un sous-groupe discret Zariski dense $\Gamma$ de $G$, démontrée à la section 2.3, pour montrer que la mesure de comptage $\sum_{\gamma \in \Gamma} \delta_{\mu(\gamma)}$, c'est-à-dire la mesure image de $\sum_{\gamma \in \Gamma} \delta_{\gamma}$ par la composante de Cartan vérifie les hypothèses des énoncés de la partie 3 . Nous commençons par contrôler l'indicateur de croissance de l'image par la composante de Cartan d'une mesure de Haar de $G$. Ensuite, nous utilisons les sous-semi-groupes libres de $\Gamma$ pour montrer que ses exposants de convergence sont nécessairement positifs. Enfin, nous appliquons les résultats de la partie 3 à l'étude de $\Gamma$. 


\subsection{Préliminaires}

\subsubsection{Estimations de volume dans $G$}

Nous commençons par calculer ici l'indicateur de croissance de la mesure image par $\mu$ d'une mesure de Haar de $G$.

On choisit une fois pour toutes une mesure de Haar $\varrho$ sur $G$. On note $\nu_{G}$ la mesure sur $E$ image de $\varrho$ par $\mu$ et

$$
\psi_{G}=\frac{\psi_{\nu_{G}}}{\log q}
$$

Montrons que $\psi_{G}=\rho$.

Supposons que $\mathbb{K}$ est $\mathbb{R}$ ou $\mathbb{C}$.

Proposition 4.1.1. ([11, I.5.8]) La mesure $\nu_{G}$ est absolument continue par rapport à la classe de la mesure de Lebesgue. Si $\lambda$ est une mesure de Lebesgue sur E, $i l$ existe un réel $c>0$ tel que, pour tout $x$ dans $E^{+}$, on ait :

$$
\frac{\mathrm{d} \nu_{G}}{\mathrm{~d} \lambda}(x)=c \prod_{\alpha \in \Sigma^{+}} \sinh (\alpha(x))^{m_{\alpha}} .
$$

Corollaire 4.1.2. Pour tout $x$ dans $E^{+}$, on a :

$$
\psi_{G}(x)=\rho(x) .
$$

Supposons que $\mathbb{K}$ est non-archimédien. Pour $z$ dans $Z^{+}$, notons $\theta_{z}$ l'ensemble des $\alpha$ dans $\Pi$ tels que $\alpha(\nu(z))>0$.

Proposition 4.1.3. ([13, 3.2.7]) Il existe une famille $\left(q_{\theta}\right)_{\theta \subset \Pi}$ de nombres réels $>0$ telle que, pour tout $z$ dans $Z^{+}$, on ait :

$$
\frac{\varrho(K z K)}{\varrho(K)}=q_{\theta_{z}} q^{\rho(\nu(z))} .
$$

Corollaire 4.1.4. Pour tout $x$ dans $E^{+}$, on $a$ :

$$
\psi_{G}(x)=\rho(x) \text {. }
$$

\subsubsection{Exposant de convergence des semi-groupes libres}

Soit $\Delta$ un semi-groupe libre de générateurs $\xi$ et $\eta$. Pour tous éléments $x$ et $y$ d'un semi-groupe $S$, on note $\Phi_{x, y}^{S}$ l'unique homomorphisme de $\Delta$ dans $S$ envoyant $\xi$ sur $x$ et $\eta$ sur $y$.

Pour calculer les exposants de convergence des sous-semi-groupes fournis par la proposition 2.2.7, nous utiliserons :

Lemme 4.1.5. Pour tous réels $u, v>1$, la série de Dirichlet

$$
\vartheta(t)=\sum_{\zeta \in \Delta} \frac{1}{\Phi_{u, v}^{\mathbb{R}_{+}^{*}(\zeta)^{t}}} \quad(t \in \mathbb{R})
$$


a pour exposant de convergence l'unique réel $\tau$ tel que

$$
\frac{1}{u^{\tau}}+\frac{1}{v^{\tau}}=1
$$

On $a \tau>0$ et, pour tout $t>\tau$,

$$
\vartheta(t)=\frac{1}{1-\left(\frac{1}{u^{t}}+\frac{1}{v^{t}}\right)} .
$$

Démonstration. Pour tout entier naturel $n$, on note $\Delta_{n}$ l'ensemble des éléments de $\Delta$ dont la longueur comme mot en $\xi$ et en $\eta$ est $\leq n$. On a :

$$
\Delta_{n+1}=\{e\} \sqcup \xi \Delta_{n} \sqcup \eta \Delta_{n} .
$$

Soit $\tau$ l'unique réel tel que

$$
\frac{1}{u^{\tau}}+\frac{1}{v^{\tau}}=1
$$

On a $\tau>0$.

On pose, pour tout entier naturel $n$ et pour tout réel $t$,

$$
\vartheta_{n}(t)=\sum_{\zeta \in \Delta_{n}} \frac{1}{\Phi_{u, v}^{\mathbb{R}_{+}^{*}}(\zeta)^{t}} .
$$

Il vient, pour tout entier naturel $n$ et pour tout réel $t$,

$$
\vartheta_{n}(t) \leq \vartheta_{n+1}(t)=1+\frac{1}{u^{t}} \vartheta_{n}(t)+\frac{1}{v^{t}} \vartheta_{n}(t)
$$

Donc, d'une part, pour tout réel $t$ tel que $\vartheta(t)<\infty$, on a :

$$
\frac{1}{u^{t}}+\frac{1}{v^{t}} \neq 1 \text { et } \vartheta(t)=\frac{1}{1-\left(\frac{1}{u^{t}}+\frac{1}{v^{t}}\right)}
$$

et, par conséquent, l'exposant de convergence de $\vartheta$ est $\geq \tau$. D'autre part, pour tout réel $t>\tau$ et pour tout entier $n \geq 1$,

$$
\vartheta_{n}(t) \leq \frac{1}{1-\left(\frac{1}{u^{t}}+\frac{1}{v^{t}}\right)}
$$

Donc $\vartheta(t)<\infty$ et l'exposant de convergence de $\vartheta$ est exactement $\tau$.

\subsection{La fonction $\psi_{\Gamma}$}

Soit $\Gamma$ un sous-groupe discret Zariski dense de $G$. On note $\nu_{\Gamma}$ la mesure

$$
\sum_{\gamma \in \Gamma} \delta_{\mu(\gamma)}
$$

C'est l'image de la mesure $\sum_{\gamma \in \Gamma} \delta_{\gamma}$ par $\mu$. Nous allons appliquer à $\nu_{\Gamma}$ la théorie générale des sections 3.2 et 3.3 . 
On note

$$
\psi_{\Gamma}=\frac{\psi_{\nu_{\Gamma}}}{\log q} \text { et } \tau_{\Gamma}=\frac{\tau_{\nu}^{\|\cdot\|}}{\log q} .
$$

Pour tout cône ouvert $\mathcal{C}$ de $E$, on note $\tau_{\mathcal{C}, \Gamma}=\frac{\tau_{\mathcal{C}, \nu_{\Gamma}}^{\|\cdot\|}}{\log q}$.

Lemme 4.2.1. Pour tout cône ouvert $\mathcal{C}$ de $E$ rencontrant $l_{\Gamma}$, on a :

$$
\tau_{\mathcal{C}, \Gamma}>0 \text {. }
$$

Rappelons qu'on a noté $F_{\Gamma}$ le sous-espace vectoriel de $E$ engendré par $l_{\Gamma}:$ si $\mathbb{K}$ est $\mathbb{R}$ et si $\mathbf{G}$ est semi-simple, $F_{\Gamma}=E$.

Démonstration. Soit $\mathcal{C}$ un cône ouvert de $E$ rencontrant $l_{\Gamma}$. L'intérieur dans $F_{\Gamma}$ de $l_{\Gamma} \cap \mathcal{C}$ est non vide. D'après la proposition 2.2 .7 , il existe un réel $\kappa \geq 0$, des éléments $\gamma_{1}$ et $\gamma_{2}$ dans $\Gamma$ et des vecteurs $x_{1}$ et $x_{2}$ dans $\mathcal{C} \cap l_{\Gamma}$ tels que le sous-semi-groupe $\Delta$ de $\Gamma$ engendré par $\gamma_{1}$ et $\gamma_{2}$ soit libre et que, avec les notations du paragraphe précédent, pour tout $\gamma$ dans $\Delta$,

$$
\mu(\gamma) \in \mathcal{C} \text { et }\left\|\mu(\gamma)-\Phi_{x_{1}, x_{2}}^{E}(\gamma)\right\| \leq \Phi_{\kappa, \kappa}^{\mathbb{R}}(\gamma) .
$$

On a alors, pour tout $\gamma$ dans $\Gamma$,

$$
\|\mu(\gamma)\| \leq \Phi_{\left\|x_{1}\right\|+\kappa,\left\|x_{2}\right\|+\kappa}^{\mathbb{R}}(\gamma)
$$

et, donc, pour tout réel $t \geq 0$,

$$
q^{-t\|\mu(\gamma)\|} \geq q^{-t \Phi_{\left\|x_{1}\right\|+\kappa,\left\|x_{2}\right\|+\kappa}^{\mathbb{R}}(\gamma)}=\left(\Phi_{q^{\left\|x_{1}\right\|+\kappa}, q^{\left\|x_{2}\right\|+\kappa}}^{\mathbb{R}^{*}}(\gamma)\right)^{-t} .
$$

D’après le lemme 4.1.5, la série de Dirichlet

$$
\sum_{\gamma \in \Delta} q^{-t\|\mu(\gamma)\|} \quad(t \in \mathbb{R})
$$

a un exposant de convergence $>0$. Comme $\mu(\Delta) \subset \mathcal{C}$, la série de Dirichlet

$$
\sum_{\substack{\gamma \in \Gamma \\ \mu(\gamma) \in \mathcal{C}}} q^{-t\|\mu(\gamma)\|} \quad(t \in \mathbb{R})
$$

a, elle aussi, un exposant de convergence strictement positif, ce qu'il fallait démontrer.

Théorème 4.2.2. Pour tout $x$ dans $E^{+}$, on $a$ :

$$
\psi_{\Gamma}(x) \leq \rho(x) .
$$

La mesure $\nu_{\Gamma}$ est à croissance concave et, donc, la fonction $\psi_{\Gamma}$ est concave et semi-continue supérieurement. L'ensemble

$$
\left\{x \in E \mid \psi_{\Gamma}(x)>-\infty\right\}
$$

est exactement le cône limite de $\Gamma$. De plus, $\psi_{\Gamma}$ est positive sur $l_{\Gamma}$ et strictement positive sur son intérieur relatif. 
Démonstration. Comme $\Gamma$ est un sous-groupe discret de $G$, d'après les lemmes 2.2.4 et 3.1.6, on a :

$$
\psi_{\Gamma} \leq \psi_{G}
$$

et, d'après les corollaires 4.1.2 et 4.1.4, pour tout $x$ dans $E^{+}$,

$$
\psi_{G}(x)=\rho(x) .
$$

En particulier, $\tau_{\Gamma}<\infty$ et, d'après le lemme 3.1.7, $\psi_{\Gamma}$ est semi-continue supérieurement.

D'après la proposition 2.3.1, la mesure $\nu_{\Gamma}$ est à croissance concave et, donc, d'après le théorème 3.2 .1 , la fonction $\psi_{\Gamma}$ est concave.

On a clairement $\psi_{\Gamma}=-\infty$ en dehors de $l_{\Gamma}$. Réciproquement, d'après le lemme 4.2.1, $\psi_{\Gamma}$ est positive sur $l_{\Gamma}$ et, puisqu'on a $\tau_{\Gamma}=\sup _{\|x\|=1} \psi_{\Gamma}(x)>0$, elle y prend des valeurs strictement positives. Comme elle est concave, elle est strictement positive sur l'intérieur relatif de $l_{\Gamma}$.

Corollaire 4.2.3. On $a$ :

$$
\frac{1}{a} \log _{q}(\operatorname{card}\{\gamma \in \Gamma \mid\|\mu(\gamma)\| \leq a\}) \underset{a \rightarrow \infty}{\longrightarrow} \tau_{\Gamma}
$$

et

$$
\operatorname{card}\{\gamma \in \Gamma \mid\|\mu(\gamma)\| \leq a\} \underset{a \rightarrow \infty}{=} O\left(a^{\operatorname{dim} F_{\Gamma}-1} q^{a \tau_{\Gamma}}\right)
$$

Démonstration. Soit $p$ un projecteur de $E$ sur $F_{\Gamma}$. Comme, d'après le théorème 2.2.6, $\mu(\Gamma)$ reste à distance bornée de $F_{\Gamma}$, la mesure $p_{*} \nu_{\Gamma}$ est encore à croissance concave et, d'après le lemme 3.1.6, elle a même indicateur de croissance que $\nu_{\Gamma}$. Le résultat est alors une conséquence de la proposition 3.3.1 appliquée à $p_{*} \nu_{\Gamma}$.

Nous pouvons enfin généraliser un résultat obtenu par P. Albuquerque ([1]) notamment dans le cas où $l_{\Gamma}-\{0\}$ était inclus dans $E^{++}$.

Pour toute norme $N$ sur $E$, notons $\tau_{\Gamma}^{N}$ pour $\frac{\tau_{\nu_{\Gamma}}^{N}}{\log q}$ et $\sigma_{\Gamma}^{N}$ pour $\frac{\sigma_{\nu_{\Gamma}}^{N}}{\log q}$. Rappelons qu'un cône fermé $\mathcal{C}$ d'un espace vectoriel réel $\mathcal{E}$ de dimension finie est dit saillant si et seulement s'il ne contient pas de sous-espace vectoriel de $\mathcal{E}$.

Corollaire 4.2.4. Si $l_{\Gamma}$ est saillant, ce qui est toujours vrai lorsque $\mathbf{G}$ est semisimple, pour toute norme $N$ sur $E$, on $a$ :

$$
\sigma_{\Gamma}^{N}=\tau_{\Gamma}^{N}
$$

En particulier, il existe alors un unique vecteur unitaire $x$ de $E^{+}$tel que les séries de Dirichlet

$$
\sum_{\gamma \in \Gamma} q^{-t\|\mu(\gamma)\|} \text { et } \sum_{\gamma \in \Gamma} q^{-t(x, \mu(\gamma))} \quad(t \in \mathbb{R})
$$

aient même exposant de convergence. Ce vecteur appartient à $l_{\Gamma}$. 
Démonstration. Il s'agit de l'application à $\nu_{\Gamma}$ des corollaires 3.3.3 et 3.3.5.

\section{Références}

[1] P. Albuquerque, Patterson-Sullivan theory in higher rank symmetric spaces, Geometric and functional analysis 9 (1999), 1-28.

[2] H. Abels, G.-A. Margulis and G.-A. Soifer, Semigroups containing proximal linear maps, Israel journal of mathematics 91 (1995), 1-30.

[3] Y. Benoist, Actions propres sur les espaces homogènes réductifs, Annals of mathematics 144 (1996), 315-347.

[4] Y. Benoist, Propriétés asymptotiques des groupes linéaires, Geometric and functional analysis 7 (1997), 1-47.

[5] Y. Benoist, Propriétés asymptotiques des groupes linéaires (II), Advanced studies in pure mathematics 26 (2000), 33-48.

[6] A. Borel, Linear algebraic groups, Graduate Texts in Mathematics 126, Springer Verlag, New York, 1991.

[7] N. Bourbaki, Éléments de mathématique, Groupes et algèbres de Lie, Chapitre VI : Systèmes de racines, Hermann, Paris 1968.

[8] F. Bruhat et J. Tits, Groupes réductifs sur un corps local, I. Données radicielles valuées, Publications mathématiques de l'IHES 41 (1972), 5-251.

[9] F. Bruhat et J. Tits, Groupes réductifs sur un corps local, II. Schémas en groupes. Existence d'une donnée radicielle valuée, Publications mathématiques de l'IHES 60 (1984), 5-184.

[10] S. Helgason, Differential geometry, Lie groups and symmetric spaces, Pure and Applied Mathematics 80, Academic Press, San Diego, 1978.

[11] S. Helgason, Groups and geometric analysis, Pure and Applied Mathematics 113, Academic Press, San Diego, 1984.

[12] J. E. Humphreys, Linear algebraic groups, Graduate Text in Mathematics 21, Springer Verlag, New York, 1981.

[13] H. Matsumoto, Analyse harmonique dans les systèmes de Tits bornologiques de type affine, Lecture Notes in Mathematics 590, Springer Verlag, Berlin, 1977.

[14] S.-J. Patterson, The limit set of a fuchsian group, Acta mathematica 136 (1976), 241-273.

[15] J.-F. Quint, Sous-groupes discrets des groupes de Lie semi-simples réels et p-adiques, thèse Université Paris VII.

[16] J.-F. Quint, Cônes limites des sous-groupes discrets d'un groupe réductif sur un corps local, Transformation groups 7 (2002), 247-266.

[17] J.-F. Quint, Mesures de Patterson-Sullivan en rang supérieur, Geometric and functional analysis 12 (2002).

[18] D. Sullivan, The density at infinity of a discrete group of hyperbolic motions, Publications mathématiques de l'IHES 50 (1979), 171-202.

[19] J. Tits, Représentations linéaires irréductibles d'un groupe réductif sur un corps quelconque, Journal für die reine und angewandte Mathematik 247 (1971), 196-220.

[20] J. Tits, Reductive groups over local fields, Proceedings of the symposium in pure mathematics of the american mathematical society 33 (1977), 29-69. 
Jean-François Quint

École Normale Supérieure

Département de Mathématiques et Applications

45, rue d'Ulm

F-75230 Paris Cedex 05

France

e-mail: quint@dma.ens.fr

(Received: November 16, 2001)

(12) To access this journal online:

(ID) http://www.birkhauser.ch 